ANALYSIS \& PDE

Volume $4 \quad$ No. $5 \quad 2011$

E. BRIAN DAVIES AND ALEXANDER PUSHNITSKI

NON-WEYL RESONANCE ASYMPTOTICS FOR QUANTUM GRAPHS 


\title{
NON-WEYL RESONANCE ASYMPTOTICS FOR QUANTUM GRAPHS
}

\author{
E. Brian Davies ANd Alexander Pushnitski
}

\begin{abstract}
We consider the resonances of a quantum graph $\mathscr{G}$ that consists of a compact part with one or more infinite leads attached to it. We discuss the leading term of the asymptotics of the number of resonances of $\mathscr{G}$ in a disc of a large radius. We call $\mathscr{G}$ a Weyl graph if the coefficient in front of this leading term coincides with the volume of the compact part of $\mathscr{G}$. We give an explicit topological criterion for a graph to be Weyl. In the final section we analyze a particular example in some detail to explain how the transition from the Weyl to the non-Weyl case occurs.
\end{abstract}

\section{Introduction}

Quantum graphs. Let $\mathscr{G}_{0}$ be a finite compact metric graph. That is, $\mathscr{G}_{0}$ has finitely many edges and each edge is equipped with coordinates (denoted $x$ ) that identify this edge with a bounded interval of the real line. We choose some subset of vertices of $\mathscr{G}_{0}$, to be called external vertices, and attach one or more copies of $[0, \infty)$, to be called leads, to each external vertex; the point 0 in a lead is thus identified with the relevant external vertex. We call the thus extended graph $\mathscr{G}$. We assume that $\mathscr{G}$ has no "tadpoles", i.e., no edge starts and ends at the same vertex; this can always be achieved by introducing additional vertices, if necessary. In order to distinguish the edges of $\mathscr{G}_{0}$ from the leads, we will call the former the internal edges of $\varphi$.

In $L^{2}(\mathscr{G})$ we consider the self-adjoint operator $H=-d^{2} / d x^{2}$ with the continuity condition and the Kirchhoff boundary condition at each vertex of $\varphi$; see Section 2 for the precise definitions. The metric graph $\mathscr{G}$ equipped with the self-adjoint operator $H$ in $L^{2}(\mathscr{G})$ is called the quantum graph. We refer to the surveys [Kuchment 2004; 2008] for a general exposition of quantum graph theory. Important earlier work on resonances of quantum graphs has been carried out by Kottos and Smilansky [2003] and Kostrykin and Schrader [1999] (see also [Kostrykin and Schrader 2006; Kostrykin et al. 2007]), but their results have little overlap with ours. For more recent progress see [Exner and Lipovský 2010; Davies et al. 2010].

If the set of leads is nonempty, it is easy to show by standard techniques (see [Ong 2006, Lemma 1], for example) that the spectrum of $H$ is $[0, \infty)$. The operator $H$ may have embedded eigenvalues.

Resonances of $\boldsymbol{H}$. The "classical" definition of resonances is this:

MSC2000: primary 34B45; secondary 35B34, 47E05.

Keywords: quantum graph, resonance, Weyl asymptotics. 
Definition 1.1. We will say that $k \in \mathbb{C}, k \neq 0$, is a resonance of $H$ (or, by a slight abuse of terminology, a resonance of $(G)$ if there exists a resonance eigenfunction $f \in L_{\mathrm{loc}}^{2}(\mathscr{G}), f \not \equiv 0$, which satisfies the equation

$$
-f^{\prime \prime}(x)=k^{2} f(x), \quad x \in \mathscr{G},
$$

on each edge and lead of $\mathscr{G}$, is continuous on $\mathscr{G}$, satisfies the Kirchhoff boundary condition at each vertex of $\mathscr{G}$ and the radiation condition

$$
f(x)=f(0) e^{i k x}
$$

on each lead of $\mathscr{G}$. We denote the set of all resonances of $H$ by $\mathscr{R}$.

Any solution to $(1-1)$ on a lead $\ell=[0, \infty)$ satisfies

$$
f(x)=\gamma_{\ell} e^{i k x}+\gamma_{\ell}^{\prime} e^{-i k x}
$$

Definition 1.1 requires that there exists a nonzero solution with all coefficients $\gamma_{\ell}^{\prime}$ vanishing. It is easy to see that all resonances must satisfy $\operatorname{Im} k \leq 0$; indeed, if $k_{0}$ with $\operatorname{Im} k_{0}>0$ is a resonance then the corresponding resonance eigenfunction is in $L^{2}(\mathscr{G})$, so $k_{0}^{2}$ is an eigenvalue of $H$, which is impossible since $k_{0}^{2} \notin[0, \infty)$. As we will only be interested in the asymptotics of the number of resonances in large disks, we exclude the case $k=0$ from further consideration. In the absence of leads, the spectrum of $H$ consists of nonnegative eigenvalues and $k \neq 0$ is a resonance if and only if $k \in \mathbb{R}$ and $k^{2}$ is an eigenvalue of $H$.

It is well known (see [Exner and Lipovský 2007; 2010], for example) that this "classical" definition of a resonance coincides with the definition via exterior complex scaling (see [Aguilar and Combes 1971; Simon 1973; Sjöstrand and Zworski 1991]). In the complex scaling approach, the resonances of $H$ are identified with the eigenvalues of an auxiliary nonselfadjoint operator $H(i \theta), \theta \in(0, \pi)$. The algebraic multiplicity of a resonance is then defined as the algebraic multiplicity of the corresponding eigenvalue of $H(i \theta)$. We discuss this in more detail in Section 2, where we show that the multiplicity is independent of $\theta$. In particular, we show (in Theorem 2.3) that any $k \in \mathbb{R}, k \neq 0$, is a resonance if and only if $k^{2}$ is an eigenvalue of $H$ and in this case the corresponding multiplicities coincide.

We define the resonance counting function by

$$
N(R)=\#\{k: k \in \mathscr{R},|k| \leq R\}, \quad R>0,
$$

with the convention that each resonance is counted with its algebraic multiplicity taken into account. Note that the set $\mathscr{R}$ of resonances is invariant under the symmetry $k \rightarrow-\bar{k}$, so this method of counting yields, roughly speaking, twice as many resonances as one would obtain if one imposed an additional condition $\operatorname{Re}(k) \geq 0$. In particular, in the absence of leads, $N(R)$ equals twice the number of eigenvalues $\lambda \neq 0$ of $H$ (counting multiplicities) with $\lambda \leq R^{2}$.

Main result. This paper is concerned with the asymptotics of the resonance counting function $N(R)$ as $R \rightarrow \infty$. We say that $G$ is a Weyl graph if

$$
N(R)=\frac{2}{\pi} \operatorname{vol}\left(\varphi_{0}\right) R+o(R), \quad \text { as } R \rightarrow \infty,
$$


where $\operatorname{vol}\left(\mathscr{G}_{0}\right)$ is the sum of the lengths of the edges of $\mathscr{G}_{0}$. If there are no leads then $H$ has pure point spectrum, resonances are identified with eigenvalues of $H$ and Weyl's law (1-2) may be proved by Dirichlet-Neumann bracketing. Thus, every compact quantum graph is Weyl in our sense. As we show below, in the presence of leads this may not be the case.

We call an external vertex $v$ of $\varphi$ balanced if the number of leads attached to $v$ equals the number of internal edges attached to $v$. If $v$ is not balanced, we call it unbalanced. Our main result is this:

Theorem 1.2. One has

$$
N(R)=\frac{2}{\pi} W R+O(1), \quad \text { as } R \rightarrow \infty,
$$

where the coefficient $W$ satisfies $0 \leq W \leq \operatorname{vol}\left(\varphi_{0}\right)$. One has $W=\operatorname{vol}\left(\varphi_{0}\right)$ if and only if every external vertex of 9 is unbalanced.

This theorem shows, in particular, that as the graph becomes larger and more complex the failure of Weyl's law becomes increasingly likely in an obvious sense.

Discussion. The simplest example of a graph $\mathscr{G}$ with a balanced external vertex occurs when exactly one lead $\ell$ and exactly one internal edge $e$ meet at a vertex. In this case, one can merge $e$ and $\ell$ into a new lead; this will not affect the resonances of $\mathscr{G}$ but will reduce vol $\mathscr{G}_{0}$. This already shows that $\mathscr{G}$ cannot be Weyl. Section 6 discusses the another simple example.

Our proof of Theorem 1.2 consists of two steps. The first step is to identify the set $\mathscr{R}$ of resonances with the set of zeros of $\operatorname{det} A(k)$, where $A(k)$ is a certain analytic matrix-valued function. This identification is straightforward, but it has a subtle aspect: this is to show that the algebraic multiplicity of a resonance coincides with the order of the zero of $\operatorname{det} A(k)$. This is done in Sections 4 and 5 by employing a range of rather standard techniques of spectral theory, including a resolvent identity which involves the Dirichlet-to-Neumann map.

The function $\operatorname{det} A(k)$ turns out to be an exponential polynomial. By a classical result (Theorem 3.2), the asymptotics of the zeros of an exponential polynomial can be explicitly expressed in terms of the coefficients of this polynomial. Thus, the second step of our proof is a direct and completely elementary analysis of the matrix $A(k)$ which allows us to relate the required information about the coefficients of the polynomial $\operatorname{det} A(k)$ to the question of whether the external vertices of $\varphi$ are balanced. This is done in Section 3.

Resonance asymptotics of Weyl type have been established for compactly supported potentials on the real line, a class of superexponentially decaying potentials on the real line, compactly supported potentials on cylinders and Laplace operators on surfaces with finite volume hyperbolic cusps in [Zworski 1987; Froese 1997; Christiansen 2004; Parnovski 1995] respectively. The proofs rely upon theorems about the zeros of certain classes of entire functions. Likewise, our analysis uses a simple classical result (Theorem 3.2) about zeros of exponential polynomials.

The situation with resonance asymptotics for potential and obstacle scattering in Euclidean space in dimensions greater than one and in hyperbolic space is more complicated and still not fully understood; the current state of knowledge is described in [Stefanov 2006; Borthwick 2010]. Here we remark only 
that generically the resonance asymptotics in the multidimensional case is not given by the Weyl formula. We hope that Theorem 1.2 can provide some insight to the multidimensional case.

One may approach the resonances of quantum graphs by studying the scattering matrix. A detailed account of resonance scattering for quantum graphs from the physics perspective and some associated numerical calculations can be found in [Kottos and Smilansky 2003]. The graphs considered in that reference have no balanced external vertices, so the non-Weyl phenomenon does not occur there. Resonances for quantum graphs have also been discussed in [Exner and Lipovský 2010]. Our paper has little technical content in common with either of those articles, in spite of their common themes.

After this paper was written the main results were extended in [Davies et al. 2010] to graphs with general self-adjoint boundary conditions at the vertices; the results there emphasise the exceptional nature of non-Weyl resonance asymptotics.

Example. In Section 6 we consider the resonances of a particularly simple quantum graph which can be described as a circle with two leads attached to it. Theorem 1.2 says that if the leads are attached at different points on the circle, the corresponding quantum graph is Weyl, and if they are attached at the same point, we have a non-Weyl graph. When the two points where the leads are attached move closer to each other and eventually coalesce, one observes the transition from the Weyl to the non-Weyl case. We study this transition in much detail. We show that as the two external vertices get closer, "half" of the resonances move off to infinity. In the course of this analysis, we also obtain bounds on the positions of individual resonances for this model.

The same example was recently considered by Exner and Lipovský [Exner and Lipovský 2010] subject to general boundary conditions that include the Kirchhoff's boundary condition case as a singular limit. Although some of their results are broadly similar to ours, none of our theorems may be found in [Exner and Lipovský 2010].

\section{Resonances via complex scaling}

Here we introduce the necessary notation, recall the definition of resonances via the complex scaling procedure and show that the resonances on the real axis coincide with the eigenvalues of $H$.

Notation. Let $E^{\text {int }}$ be the set of all internal edges of $\varphi$ (i.e., the set of all edges of $\varphi_{0}$ ) and let $E^{\text {ext }}$ be the set of all leads; we also denote $E=E^{\text {int }} \cup E^{\text {ext }}$. The term "edge" without an adjective will refer to any element of $E$. For $e \in E^{\text {int }}$, we denote by $\rho(e)$ the length of $e$; i.e., an edge $e \in E^{\text {int }}$ is identified with the interval $[0, \rho(e)]$.

Let $V$ be the set of all vertices of $\varphi$, let $V^{\text {ext }}$ be the set of all external vertices, and let $V^{\text {int }}=V \backslash V^{\text {ext }}$; the elements of $V^{\text {int }}$ will be called internal vertices. The degree of a vertex $v$ is denoted by $d(v)$. The number of leads attached to an external vertex $v$ is denoted by $q(v)$; we also set $q(v)=0$ for $v \in V^{\text {int }}$.

If an edge or a lead $e$ is attached to a vertex $v$, we write $v \in e$. If two vertices $u, v$ are connected by one or more edges, we write $u \sim v$.

We denote by $\mathscr{G}_{\infty}$ the graph $\varphi_{\text {with }}$ all the internal edges and vertices removed. We let $\chi_{0}$ and $\chi_{\infty}$ be the characteristic functions of $\mathscr{G}_{0}$ and $\mathscr{G}_{\infty}$. 
Let $f: G \rightarrow \mathbb{C}$ be a function such that the restriction of $f$ onto every edge is continuously differentiable. Then for $v \in V$, we denote by $N_{v} f$ the sum of the outgoing derivatives of $f$ at $v$ over all edges attached to $v$. If $v$ is an external vertex, we denote by $N_{v}^{\text {int }} f$ (resp. $N_{v}^{\text {ext }} f$ ) the sum of all outgoing derivatives of $f$ at $v$ over all internal edges (resp. leads) attached to $v$.

Let $\widetilde{C}(\mathscr{G})$ be the class of functions $f: \mathscr{G} \rightarrow \mathbb{C}$ which are continuous on $\mathscr{G} \backslash V^{\mathrm{ext}}$ and such that for each external vertex $v$ the function $f(x)$ approaches a limiting value (to be denoted by $D_{v}^{\text {int }} f$ ) as $x$ approaches $v$ along any internal edge and $f(x)$ approaches another limiting value (to be denoted by $D_{v}^{\text {ext }} f$ ) as $x$ approaches $v$ along any lead.

For any finite set $A$, we denote by $|A|$ the number of elements of $A$. We will use the identity

$$
\sum_{v \in V} d(v)=2\left|E^{\mathrm{int}}\right|+\left|E^{\mathrm{ext}}\right| .
$$

Finally, we use the notation $\mathbb{C}_{+}=\{z \in \mathbb{C}: \operatorname{Im} z>0\}$.

The operator $\boldsymbol{H}(\varkappa)$. The domain of the self-adjoint operator $H$ consists of all continuous functions $f: \mathscr{G} \rightarrow \mathbb{C}$ such that the restriction of $f$ onto any $e \in E$ lies in the Sobolev space $W_{2}^{2}(e)$, and $f$ satisfies the Kirchhoff boundary condition $N_{v} f=0$ on every vertex $v$ of $\mathscr{G}$.

For $\varkappa \in \mathbb{R}$, let $U(\varkappa): L^{2}(\varphi) \rightarrow L^{2}(\mathscr{G})$ be the unitary operator which acts as identity on $L^{2}\left(\varphi_{0}\right)$ and as a dilation on all leads $\ell=[0, \infty)$ :

$$
(U(\varkappa) f)(x)=e^{\varkappa / 2} f\left(e^{\varkappa} x\right), \quad x \in \ell .
$$

Note that $U(\varkappa)^{*}=U(-\varkappa)$ for any $\varkappa \in \mathbb{R}$. Consider the operator

$$
H(\varkappa)=U(\varkappa) H U(-\varkappa) .
$$

It admits an analytic continuation to $\varkappa \in \mathbb{C}$, which we describe below.

Definition 2.1. For $\varkappa \in \mathbb{C}$, the operator $H(\varkappa)$ in $L^{2}(\varphi)$ acts according to the formula

$$
(H(x) f)(x)= \begin{cases}-f^{\prime \prime}(x) & \text { if } x \in \mathscr{G}_{0}, \\ -e^{-2 \varkappa} f^{\prime \prime}(x) & \text { if } x \in \mathscr{G}_{\infty} .\end{cases}
$$

The domain of $H(\varkappa)$ is defined to be the set of all $f: \mathscr{G} \rightarrow \mathbb{C}$ which satisfy the following conditions:

(i) The restriction of $f$ onto any $e \in E$ lies in the Sobolev space $W_{2}^{2}(e)$.

(ii) $f \in \widetilde{C}(\varphi)$.

(iii) $f$ satisfies the condition $N_{v} f=0$ at every internal vertex $v$.

(iv) For any $v \in V^{\text {ext }}$, one has

$$
\begin{aligned}
D_{v}^{\text {int }} f-e^{-\varkappa / 2} D_{v}^{\text {ext }} f & =0, \\
N_{v}^{\text {int }} f+e^{-3 \varkappa / 2} N_{v}^{\text {ext }} f & =0 .
\end{aligned}
$$


In particular, $H(0)$ is the operator called $H$ so far. For complex $\varkappa$, the operator $H(x)$ is in general nonselfadjoint. A standard straightforward computation shows that for any $\varkappa \in \mathbb{C}$ the operator $H(\varkappa)$ is closed and

$$
H(\varkappa)^{*}=H(\bar{\varkappa}) .
$$

Resonances via complex scaling. The following theorem is standard in the method of complex scaling; see [Aguilar and Combes 1971; Simon 1973; Sjöstrand and Zworski 1991; Exner and Lipovský 2007]:

Theorem 2.2. The family of operators $H(\varkappa), \varkappa \in \mathbb{C}$, is analytic in the sense of Kato (see, for example, [Reed and Simon 1978, Section XII.2]), and

$$
H\left(\varkappa+\varkappa_{0}\right)=U\left(\varkappa_{0}\right) H(\varkappa) U\left(-\varkappa_{0}\right) \quad \text { for all } \varkappa \in \mathbb{C} \text { and all } \varkappa_{0} \in \mathbb{R} .
$$

The essential spectrum of $H(\varkappa)$ coincides with the half-line $e^{-2 \varkappa}[0, \infty)$. Let $\theta \in(0, \pi)$; then the sector $0<\arg \lambda<2 \pi-2 \theta, \lambda \neq 0$, contains no eigenvalues of $H(i \theta)$, and any $\lambda \neq 0$ in the sector $2 \pi-2 \theta<$ $\arg \lambda \leq 2 \pi$ is an eigenvalue of $H(i \theta)$ if and only if $\lambda=k^{2}$ with $k \in \mathscr{R}$.

For completeness, we give the proof in Section 5.

As $\theta \in(0, \pi)$ increases monotonically, the essential spectrum $e^{-2 i \theta}[0, \infty)$ of $H(i \theta)$ rotates clockwise, uncovering more and more eigenvalues $\lambda$. These eigenvalues are identified with the resonances $k$ of $H$ via $\lambda=k^{2}$. If $\lambda \neq 0$ is an eigenvalue of $H(i \theta), \theta \in(0, \pi), 2 \pi-2 \theta<\arg \lambda \leq 2 \pi$, Kato's theory of analytic perturbations implies that the eigenvalue and associated Riesz spectral projection depend analytically on $\theta$. Noting (2-8) and using analytic continuation it follows that the algebraic multiplicity of $\lambda$ is independent of $\theta$. It is easy to see directly that the geometric multiplicity of $\lambda$ is also independent of $\theta$. The algebraic (resp. geometric) multiplicity of a resonance $k$ is defined as the algebraic (resp. geometric) multiplicity of the corresponding eigenvalue $\lambda=k^{2}$ of $H(i \theta)$.

Resonances on the real line. The geometric multiplicities of resonances will not play any role in our analysis. However, we note that for the Schrödinger operator on the real line, resonances can have arbitrary large algebraic multiplicity [Korotyaev 2005], while their geometric multiplicity is always equal to one. This gives an example of resonances with distinct algebraic and geometric multiplicities. It would be interesting to see if one can have distinct algebraic and geometric multiplicities of resonances for quantum graphs in the situation we are discussing. We have nothing to say about this except for the case of the resonances on the real line:

Theorem 2.3. (i) If $k \in \mathbb{R}, k \neq 0$, is a resonance of $H$ then the algebraic and geometric multiplicities of $k$ coincide.

(ii) Any $k \in \mathbb{R}, k \neq 0$, is a resonance of $H$ if and only if $k^{2}$ is an eigenvalue of $H$ and the multiplicity of the resonance $k$ coincides with the multiplicity of the eigenvalue $k^{2}$.

Proof. 1. Let $\lambda>0$ be an eigenvalue of $H$ with the eigenfunction $f$. If $\ell=[0, \infty)$ is a lead, then $f(x)=\gamma_{\ell} e^{i k x}+\gamma_{\ell}^{\prime} e^{-i k x}, x \in \ell$, where $k^{2}=\lambda$. Since $f \in L^{2}(\ell)$, we conclude that $\gamma_{\ell}=\gamma_{\ell}^{\prime}=0$ and so 
$f \equiv 0$ on all leads. It follows that $f \in \operatorname{Dom} H(i \theta)$ for all $\theta$ and $H(i \theta) f=\lambda f$. This argument proves that

$$
\operatorname{dim} \operatorname{Ker}(H(i \theta)-\lambda I) \geq \operatorname{dim} \operatorname{Ker}(H-\lambda I) .
$$

2. Let $f \in \operatorname{Ker}(H(i \theta)-\lambda I), \lambda>0, \theta \in(0, \pi)$. Let us prove that $f$ vanishes identically on all leads. Let $\lambda=k^{2}, k>0$. On every lead, we have

$$
f(x)=f(0) \exp \left(i e^{i \theta} k x\right) .
$$

Consider the difference

$$
\omega(f)=\int_{\varphi_{0}}\left|f^{\prime}(x)\right|^{2} d x-\lambda \int_{\varphi_{0}}|f(x)|^{2} d x=\int_{\varphi_{0}}\left|f^{\prime}(x)\right|^{2} d x+\int_{\varphi_{0}} f^{\prime \prime}(x) \overline{f(x)} d x .
$$

Integrating by parts, we get

$$
\omega(f)=-\sum_{v \in V^{\mathrm{ext}}}\left(N_{v}^{\mathrm{int}} f\right) \overline{D_{v}^{\mathrm{int}} f} .
$$

Using the boundary condition (2-5) and formula (2-10), we obtain

$$
\omega(f)=i k \sum_{v \in V^{\mathrm{ext}}}\left|D_{v}^{\mathrm{ext}} f\right|^{2} q(v) .
$$

By the definition (2-11) of $\omega(f)$, we have $\operatorname{Im} \omega(f)=0$. This yields that $\left|D_{v}^{\text {ext }} f\right|=0$ on all external vertices $v$. By (2-10), it follows that $f$ vanishes identically on all leads.

3. By combining the previous step of the proof with (2-5) and (2-6) we obtain $D_{v}^{\text {int }} f=N_{v}^{\text {int }} f=0$. It follows that for any $f \in \operatorname{Ker}(H(i \theta)-\lambda I), \lambda>0, \theta \in(0, \pi)$, we have $f \in \operatorname{Dom} H$ and $H f=\lambda f$. This argument also proves that

$$
\operatorname{dim} \operatorname{Ker}(H-\lambda I) \geq \operatorname{dim} \operatorname{Ker}(H(i \theta)-\lambda I) .
$$

4. It remains to prove that if $\lambda>0$ is an eigenvalue of $H(i \theta), \theta \in(0, \pi)$, then its algebraic and geometric multiplicities coincide. Suppose this is not the case. Then there exist nonzero elements $f, g \in \operatorname{Dom} H(i \theta)$ such that $H(i \theta) g=\lambda g$ and $(H(i \theta)-\lambda I) f=g$.

By step 2 of the proof, $g$ vanishes on all leads. It follows that on all leads the function $f$ satisfies (2-10). Next, since $g(x)=-f^{\prime \prime}(x)-\lambda f(x)$ on $\mathscr{G}_{0}$, we have

$$
0<\int_{\varphi_{0}}|g(x)|^{2} d x=-\int_{\varphi_{0}}\left(f^{\prime \prime}(x)+\lambda f(x)\right) \overline{g(x)} d x .
$$

Integrating by parts, we get

$$
\begin{aligned}
& -\int_{\mathscr{G}_{0}}\left(f^{\prime \prime}(x)+\lambda f(x)\right) \overline{g(x)} d x \\
& \quad=-\int_{\mathscr{G}_{0}} f(x)\left(\overline{g^{\prime \prime}(x)}+\lambda \overline{g(x)}\right) d x+\sum_{v \in V^{\mathrm{ext}}}\left(N_{v}^{\mathrm{int}} f\right)\left(\overline{D_{v}^{\text {int }} g}\right)-\sum_{v \in V^{\mathrm{ext}}}\left(D_{v}^{\mathrm{int}} f\right)\left(\overline{N_{v}^{\text {int }} g}\right) .
\end{aligned}
$$


Consider the three terms in the right-hand side of (2-14). The first term vanishes since $H(i \theta) g=\lambda g$. Next, since $g \equiv 0$ on $\mathscr{G}_{\infty}$, we have $D_{v}^{\text {ext }} g=N_{v}^{\text {ext }} g=0$ for any $v \in V^{\text {ext }}$. By the boundary conditions (2-5) and (2-6) for $g$ it follows that $D_{v}^{\text {int }} g=N_{v}^{\text {int }} g=0$. Thus, the second and third terms in the right-hand side of (2-14) also vanish. This contradicts (2-13).

\section{Proof of Theorem 1.2}

Here we describe the resonances as zeros of $\operatorname{det} A(k)$, where $A(k)$ is certain entire matrix-valued function. Using this characterisation, we prove our main result.

Definition of $\boldsymbol{A}(\boldsymbol{k})$. Fix $k \in \mathbb{C}_{+}$. Let $\mathscr{L}(k)$ denote the space of all solutions $f \in L^{2}(\mathscr{G})$ to $-f^{\prime \prime}=k^{2} f$ on $\mathscr{G}$ without any boundary conditions. The restriction of $f \in \mathscr{L}(k)$ to any internal edge $e$ has the form $f_{e}(x)=\alpha_{e} e^{i k x}+\beta_{e} e^{-i k x}$, and the restriction of $f$ to any lead $\ell$ has the form $f_{\ell}(x)=\gamma_{\ell} e^{i k x}$. Thus, $\operatorname{dim} \mathscr{L}(k)=2\left|E^{\mathrm{int}}\right|+\left|E^{\mathrm{ext}}\right|$.

Let us describe in detail the set of all conditions on $f \in \mathscr{L}(k)$ required to ensure that $f$ is a resonance eigenfunction. If $f_{e}$ denotes the restriction of $f$ to an edge $e$, then we can write the continuity conditions at the vertex $v$ as

$$
f_{e}(v)=\zeta_{v} \text { for all } e \ni v,
$$

where $\zeta_{v} \in \mathbb{C}$ is an auxiliary variable. We also have the condition

$$
N_{v} f=0, \quad v \in V .
$$

Writing down conditions (3-1), (3-2) for every vertex $v \in V$, we obtain

$$
N=\sum_{v \in V} d(v)+|V|=2\left|E^{\mathrm{int}}\right|+\left|E^{\mathrm{ext}}\right|+|V|
$$

conditions. Our variables are $\zeta_{v}, \alpha_{e}, \beta_{e}, \gamma_{\ell}$; altogether we have

$$
|V|+\operatorname{dim} \mathscr{L}(k)=|V|+2\left|E^{\mathrm{int}}\right|+\left|E^{\mathrm{ext}}\right|=N
$$

variables. Let $\zeta, \alpha, \beta, \gamma$ be the sequences of coordinates $\zeta_{v}, \alpha_{e}, \beta_{e}, \gamma_{\ell}$ of length $|V|,\left|E^{\text {int }}\right|,\left|E^{\text {int }}\right|$, $\left|E^{\text {ext }}\right|$ respectively, and let $v=(\zeta, \alpha, \beta, \gamma)^{\top} \in \mathbb{C}^{N}$. We may write the constraints (3-1), (3-2) in the form $A v=0$, where $A$ is an $N \times N$ matrix. Each row of $A$ relates to one of the constraints, and each constraint is of the form

$$
y \cdot \zeta+a \cdot \alpha+b \cdot \beta+g \cdot \gamma=0 .
$$

If the constraint is of the form (3-2), then $y=0$ and $a, b, g$ all contain a multiplicative factor $i k$ which we eliminate before proceeding. The coefficient $a_{e}$ is $0, \pm 1$, or $\pm e^{i k \rho(e)}$, and the coefficient $b_{e}$ is 0 , \pm 1 , or $\pm e^{-i k \rho(e)}$. The coefficient $g_{\ell}$ is 0 or 1 , and the coefficient $y_{v}$ is 0 or -1 .

We have not specified the order of the rows or columns of $A(k)$. However, the object of importance in the sequel is the set of zeros of $\operatorname{det} A(k)$, and the choice of the order of rows or columns of $A(k)$ will not affect this set. 
Example. As an example, let us display the matrix $A(k)$ for a graph which consists of two vertices $v_{1}$ and $v_{2}$, two edges $e_{1}$ and $e_{2}$ of length $\rho_{1}$ and $\rho_{2}$ and a lead attached at $v_{1}$. In this case we have, with $z_{j}=e^{i k \rho_{j}}$,

$$
A(k)=\left(\begin{array}{rcccccc}
0 & 0 & z_{1} & z_{2} & -z_{1}^{-1} & -z_{2}^{-1} & 0 \\
0 & 0 & 1 & 1 & -1 & -1 & 1 \\
-1 & 0 & 0 & 0 & 0 & 0 & 1 \\
-1 & 0 & 1 & 0 & 1 & 0 & 0 \\
-1 & 0 & 0 & 1 & 0 & 1 & 0 \\
0 & -1 & z_{1} & 0 & z_{1}^{-1} & 0 & 0 \\
0 & -1 & 0 & z_{2} & 0 & z_{2}^{-1} & 0
\end{array}\right) .
$$

Resonances as zeros of $\operatorname{det} \boldsymbol{A}(\boldsymbol{k})$. Although $A(k)$ was defined for $k \in \mathbb{C}_{+}$, we see that all elements of $A(k)$ are entire functions of $k \in \mathbb{C}$. Thus, we will consider $A(k)$ as an entire matrix-valued function of $k$.

In Sections 4 and 5 we prove:

Theorem 3.1. Any $k_{0} \neq 0$ is a resonance of $H$ if and only if $\operatorname{det} A\left(k_{0}\right)=0$. In this case, the algebraic multiplicity of the resonance $k_{0}$ coincides with the order of $k_{0}$ as a zero of $\operatorname{det} A(k)$.

The first part of this theorem is obvious: by the construction of the matrix $A$, we have $\operatorname{det} A\left(k_{0}\right)=0$ iff there exists a nonzero resonance eigenfunction $f \in \mathscr{L}\left(k_{0}\right)$. The part concerning multiplicity is less obvious. Unfortunately, we were not able to find a completely elementary proof of this part. The proof we give in Sections 4-5 involves a standard set of techniques from the spectral theory of quantum graphs: a resolvent identity involving the Dirichlet-to-Neumann map and a certain trace formula.

By Theorem 3.1, the question reduces to counting the total multiplicity of zeros of the entire function det $A(k)$ in large discs. As is clear from the structure of the matrix $A(k)$, its determinant is an exponential polynomial, i.e., a linear combination of the terms of the type $e^{i \sigma k}, \sigma \in \mathbb{R}$. Thus, we need to discuss the zeros of exponential polynomials.

Zeros of exponential polynomials. Exponential polynomials are entire functions $F(k), k \in \mathbb{C}$, of the form

$$
F(k)=\sum_{r=1}^{n} a_{r} \mathrm{e}^{i \sigma_{r} k},
$$

where $a_{r}, \sigma_{r} \in \mathbb{C}$ are constants. The study of the zeros of such polynomials has a long history; see, e.g., [Langer 1931] and references therein. For more recent literature see [Moreno 1973]. Some of these results were rediscovered in [Davies 2003; Davies and Incani 2010; Incani 2009], where they were used to analyze the spectra of nonselfadjoint systems of ODEs and directed finite graphs. The asymptotic distribution of the zeros of $F$ depends heavily on the location of the extreme points of the convex hull of the set $\cup_{r=1}^{n}\left\{\sigma_{r}\right\}$.

We are only interested in the case in which $\sigma_{r}$ are distinct real numbers. We set $\sigma^{-}=\min \left\{\sigma_{1}, \ldots, \sigma_{n}\right\}$ and $\sigma^{+}=\max \left\{\sigma_{1}, \ldots, \sigma_{n}\right\}$. For $R>0$ we denote the number of zeros of $F$ (counting their orders) in the disc $\{k \in \mathbb{C}:|k|<R\}$ by $N(R ; F)$. The following classical statement is from [Langer 1931, Theorem 3]. 
Theorem 3.2. Let $F$ be a function of the form (3-5), where $a_{r}$ are nonzero complex numbers and $\sigma_{r}$ are distinct real numbers. Then there exists a constant $K<\infty$ such that all the zeros of $F$ lie within a strip of the form $\{k:|\operatorname{Im}(z)| \leq K\}$. The counting function $N(R ; F)$ satisfies

$$
N(R ; F)=\frac{\sigma^{+}-\sigma^{-}}{\pi} R+O(1) \text { as } R \rightarrow+\infty .
$$

Estimate for $\boldsymbol{N}(\boldsymbol{R} ; \boldsymbol{F})$. Here we prove the first part of the main Theorem 1.2. Let $F(k)=\operatorname{det} A(k)$. From the structure of $A(k)$ it is clear that $F(k)$ is given by (3-5) where $a_{r}, \sigma_{r}$ are real coefficients. By Theorem 3.2, it suffices to prove that in the representation (3-5) we have

$$
\sigma^{+} \leq \operatorname{vol}\left(\mathscr{G}_{0}\right), \quad \sigma^{-} \geq-\operatorname{vol}\left(\mathscr{G}_{0}\right)
$$

In order to prove (3-6), let us discuss the entries of $A(k)$ in detail. For simplicity of notation we will not draw attention in our equations to the fact that all of the matrices below depend on $k$.

The matrix $A$ has some constant terms and some terms that are exponential in $k$. The term $e^{i k \rho(e)} \operatorname{can}$ only appear in the column associated with the variable $\alpha_{e}$ and the term $e^{-i k \rho(e)}$ can only appear in the column associated with the variable $\beta_{e}$. The columns associated with the variables $\zeta$ and $\gamma$ contain only constant terms. Since the determinant is formed from the products of entries of $A$ where every column contributes one entry to each product, we see that the maximum possible value for the coefficient $\sigma_{r}$ in (3-5) is attained when every column corresponding to the variable $\alpha_{e}$ contributes the term $e^{i k \rho(e)}$ and every column corresponding to $\beta_{e}$ contributes a constant term to the product. The maximal value of $\sigma_{r}$ thus attained will be exactly $\sum_{e \in E^{\text {int }}} \rho(e)=\operatorname{vol} \mathscr{G}_{0}$. This proves the first inequality in (3-6). The second one is proven in the same way by considering the minimal possible value for $\sigma_{r}$.

Of course, the coefficients $a^{ \pm}$of the terms $e^{ \pm i k \operatorname{vol}\left(\mathscr{G}_{0}\right)}$ in the representation (3-5) for det $A$ may well happen to be zero. Theorem 1.2 will be proven if we show that these coefficients do not vanish if and only if every external vertex of $\varphi$ is unbalanced. In what follows, for an exponential polynomial $F$ with the representation (3-5) we denote by $a^{ \pm}(F)$ the coefficient $a_{r}$ of the term $e^{i \sigma_{r} k}, \sigma_{r}= \pm \operatorname{vol}\left(\varphi_{0}\right)$.

Invariance of resonances with respect to a change of orientation. Before proceeding with the proof, we need to discuss a minor technical point. Our definition of the matrix $A(k)$ assumes that a certain orientation of all internal edges of $\mathscr{G}$ is fixed. Suppose we have changed the parametrization of an internal edge $e$ by reversing its orientation. In other words, suppose that instead of the variable $x \in[0, \rho(e)]$ we decided to use the variable $x^{\prime}=\rho(e)-x$. We claim that this change will not affect the zeros of det $A(k)$.

Indeed, let $A^{\prime}(k)$ be the matrix corresponding to the new parametrization. The matrix $A^{\prime}(k)$ corresponds to the parametrization of solutions $f \in \mathscr{L}(k)$ on $e$ by $f(x)=\alpha_{e}^{\prime} e^{i k x^{\prime}}+\beta_{e}^{\prime} e^{-i k x^{\prime}}$ instead of $\alpha_{e} e^{i k x}+\beta_{e} e^{-i k x}$. We have

$$
\left(\begin{array}{c}
\alpha_{e}^{\prime} \\
\beta_{e}^{\prime}
\end{array}\right)=\left(\begin{array}{cc}
0 & e^{-i k \rho(e)} \\
e^{i k \rho(e)} & 0
\end{array}\right)\left(\begin{array}{c}
\alpha_{e} \\
\beta_{e}
\end{array}\right), \quad \operatorname{det}\left(\begin{array}{cc}
0 & e^{-i k \rho(e)} \\
e^{i k \rho(e)} & 0
\end{array}\right)=-1,
$$

and thus $\operatorname{det} A^{\prime}(k)=-\operatorname{det} A(k)$. 
Proof of Theorem 1.2: the balanced case. Assume that a particular external vertex $v$ of $\varphi$ is balanced. Below we prove that the coefficient $a^{+}(\operatorname{det} A)$ vanishes.

Let us reorder the rows and columns of $A$ by reference to the vertex $v$. We assume that $q$ internal edges and $q$ leads are attached to $v, q \geq 2$. (The case $q=1$ is trivial because one may then merge the lead with the edge to which it is connected.) Using the invariance of resonances with respect to a change of orientation (page 738), we can choose an orientation of these internal edges so that they all end at $v$ (i.e., $v$ is identified with the point $\rho(e)$ of the intervals $[0, \rho(e)])$. Let the first $2 q$ rows of $A$ be those relating to the conditions (3-1) for the vertex $v$ and let the $(2 q+1)$-st row be the one relating to the condition (3-2) for the vertex $v$. The ordering of the remaining rows does not matter. Let the first $2 q$ columns be related to the variables $\gamma_{1}, \ldots, \gamma_{q}, \alpha_{1}, \ldots, \alpha_{q}$ and let the $(2 q+1)$-st column be related to the variable $\zeta_{v}$; see the definition of the matrix $A(k)$ in Section 3. The ordering of the remaining columns does not matter.

We write $A$ in the block form

$$
A=\left(\begin{array}{ll}
B & C \\
D & E
\end{array}\right)
$$

where $B$ is a $(2 q+1) \times(2 q+1)$ matrix. For example, in the case $q=2$ we have

$$
B=\left(\begin{array}{rrrrr}
1 & 0 & 0 & 0 & -1 \\
0 & 1 & 0 & 0 & -1 \\
0 & 0 & z_{1} & 0 & -1 \\
0 & 0 & 0 & z_{2} & -1 \\
1 & 1 & -z_{1} & -z_{2} & 0
\end{array}\right),
$$

where $z_{r}=e^{i k \rho\left(e_{r}\right)}$.

The determinant is the sum of the products of entries of $A$ where every column contributes one entry to each product. In order for the product to be of the type $a_{+} e^{i k \operatorname{vol}\left(\varphi_{0}\right)}$, each column corresponding to a variable $\alpha_{e}$ must contribute the entry $e^{i k \rho(e)}$. Thus, the constant entries of the columns corresponding to the variables $\alpha_{e}$ are irrelevant to our question and can be replaced by zeros; this will not affect the value of $a^{+}(\operatorname{det} A)$. Noticing that the columns of $D$ corresponding to the variables $\gamma_{1}, \ldots, \gamma_{q}$ and $\zeta_{v}$ are all zeros, we conclude that

$$
a^{+}(\operatorname{det} A)=a^{+}\left(\operatorname{det} A_{0}\right), \quad \text { where } A_{0}=\left(\begin{array}{cc}
B & C \\
0 & E
\end{array}\right) .
$$

By a general matrix identity, det $A_{0}=\operatorname{det} B \operatorname{det} E$. Finally, a simple row reduction shows that $\operatorname{det} B=0$; this is easy to see in the case of (3-8). Thus, the coefficient $a^{+}(\operatorname{det} A)$ vanishes. By (3-6), it follows that $\sigma^{+}<\operatorname{vol} \mathscr{G}_{0}$, as claimed.

We note (although this is not needed for our proof) that $\sigma^{-}=-\operatorname{vol} \varphi_{0}$ both in the balanced and in the unbalanced case; this will be clear from the next part of the proof.

Proof of Theorem 1.2: the unbalanced case. Assume that all external vertices are unbalanced. We will prove that

$$
\sigma^{+}=\operatorname{vol}\left(\mathscr{G}_{0}\right), \quad \sigma^{-}=-\operatorname{vol}\left(\mathscr{\varphi}_{0}\right) .
$$


The proof uses the same reduction as (3-7), but the details are somewhat more complicated, since now we have to consider all external vertices.

We label the external vertices by $v_{1}, \ldots, v_{m}$, where $m=\left|V^{\text {ext }}\right|$. For $r=1,2, \ldots, m$, let $\varphi_{r}$ denote the graph obtained from $\mathscr{G}_{0}$ by adding all the leads of $\mathscr{G}_{\text {that }}$ have ends in the set $\left\{v_{1}, \ldots, v_{r}\right\}$, so that $\mathscr{G}_{m}=\mathscr{G}_{\text {. Let }} A_{r}$ denote the constraint matrix $A$ corresponding to the graph $\mathscr{G}_{r}$ and let $a_{r}^{ \pm}=a^{ \pm}\left(\operatorname{det} A_{r}\right)$.

By the previous reasoning, the graph $\varphi_{r}$ is Weyl if and only if $a_{r}^{+} \neq 0$ and $a_{r}^{-} \neq 0$. Our claim (3-9) follows inductively from the following statements:

1. The graph $\mathscr{G}_{0}$ is Weyl.

2. The coefficient $a_{r}^{-}$is nonzero for all $r$.

3. For all $r$, if $a_{r-1}^{+} \neq 0$ then $a_{r}^{+} \neq 0$.

Item 1 holds because the operator $H$ on $\mathscr{G}_{0}$ has discrete spectrum and no other resonances. The eigenvalues obey the Weyl law by a standard variational argument using Dirichlet-Neumann bracketing.

Let us prove item 3. We reorder the rows and columns of $A_{r}$ with reference to $v_{r}$ as in the balanced case (see previous page). We assume that $p$ internal edges $e_{1}, \ldots, e_{p}$ and $q$ leads $\ell_{1}, \ldots, \ell_{q}$ are attached to $v_{r}$, and $q \neq p$. The first $q+p+1$ columns of $A_{r}$ are those relating to the variables $\gamma_{1}, \ldots, \gamma_{q}$ (associated with $\left.\ell_{1}, \ldots, \ell_{q}\right), \alpha_{1}, \ldots, \alpha_{p}$ (associated with $e_{1}, \ldots, e_{p}$ ), and $\zeta_{r}$. The first $q+p+1$ rows of $A_{r}$ are those relating to the conditions (3-1) and (3-2) for the vertex $v_{r}$. As in the balanced case, this allows us to write

$$
A_{r}=\left(\begin{array}{cc}
B_{r} & C_{r} \\
D_{r} & E_{r}
\end{array}\right)
$$

where $B_{r}$ is a $(q+p+1) \times(q+p+1)$ matrix. Writing the matrix $A_{r-1}$ in the same way with reference to the same vertex $v_{r}$, we obtain

$$
A_{r-1}=\left(\begin{array}{cc}
\widetilde{B}_{r-1} & \widetilde{C}_{r-1} \\
\widetilde{D}_{r-1} & E_{r}
\end{array}\right)
$$

where $\widetilde{B}_{r-1}$ is a $(p+1) \times(p+1)$ matrix. In other words, $\widetilde{B}_{r-1}, \widetilde{C}_{r-1}, \widetilde{D}_{r-1}$ are the matrices $B_{r}$, $C_{r}, D_{r}$ with relevant $q$ rows and $q$ columns deleted. The deleted columns correspond to the variables $\gamma_{1}, \ldots, \gamma_{q}$, and the deleted rows correspond to the conditions (3-1) associated with the leads $\ell_{1}, \ldots, \ell_{q}$. Note that the matrix $E_{r}$ is the same in (3-10) and (3-11).

Next, just as in the argument used in the balanced case, we notice that

$$
a_{r}^{+}=a^{+}\left(\operatorname{det} B_{r} \operatorname{det} E_{r}\right) \quad \text { and } \quad a_{r-1}^{+}=a^{+}\left(\operatorname{det} \widetilde{B}_{r-1} \operatorname{det} E_{r}\right) .
$$

Finally, by a simple row reduction we obtain

$$
\begin{aligned}
\operatorname{det} B_{r} & =(q-p) z_{1} \ldots z_{p}, \\
\operatorname{det} \widetilde{B}_{r-1} & =(-p) z_{1} \ldots z_{p},
\end{aligned}
$$

where $z_{j}=e^{i k \rho\left(e_{j}\right)}$. It follows that $a_{r}^{+}$and $a_{r-1}^{+}$differ by a nonzero coefficient $(p-q) / p$. This proves item 3 . 
Let us prove item 2. Here the argument follows that of the proof of item 3, only instead of keeping track of the coefficient of $e^{i k \operatorname{vol}\left(\varphi_{0}\right)}$ we need to keep track of the coefficient of $e^{-i k \operatorname{vol}\left(\mathscr{G}_{0}\right)}$, and instead of the variables $\alpha_{1}, \ldots, \alpha_{p}$ we consider the variables $\beta_{1}, \ldots, \beta_{p}$. Instead of the coefficient $(q-p)$ in (3-12) we get $(q+p)$, which never vanishes (even if $v_{r}$ is balanced). This proves our claim.

\section{A resolvent identity and its consequences}

To complete the proof of Theorem 1.2, it remains to provide the proof of Theorem 3.1. Theorem 4.2 below provides an explicit formula for the difference of the resolvents of $H(\varkappa)$ and an auxiliary operator $H_{D}(\varkappa)$; this formula is given in terms of the Dirichlet-to-Neumann map. This leads immediately to the trace formula (4-13), which is the key to our proof of Theorem 3.1 in Section 5. The formulae obtained in this section are "complex-scaled" versions of resolvent identities well known in the theory of boundary value problems; see, for example, [Gesztesy et al. 2009; 2007]

Dirichlet-to-Neumann map. Throughout this section, we assume that the parameter $k \in \mathbb{C}_{+}$is fixed. Let $\mathscr{L}(k)$ be as defined on page 736 and let $M(k)=\mathscr{L}(k) \cap C(\mathscr{G})$. Each $f \in M(k)$ determines a vector $\zeta \in \mathbb{C}^{|V|}$ by restriction to $V$. Conversely, every $\zeta \in \mathbb{C}^{|V|}$ arises from a function $f \in M(k)$; this can be seen by comparing $\operatorname{dim} \mathscr{L}(k)$ with the number of constraints imposed by writing

$$
f(v)=\zeta_{v}, \quad v \in V
$$

Finally, the assumption $k \in \mathbb{C}_{+}$implies that only one function $f \in \mathcal{M}(k)$ corresponds to each set of values $\zeta \in \mathbb{C}^{|V|}$ (otherwise we would have a complex eigenvalue of the operator with Dirichlet boundary conditions on all vertices). This shows that we may define the Dirichlet-to-Neumann map

$$
\Lambda(k): \mathbb{C}^{|V|} \rightarrow \mathbb{C}^{|V|}
$$

by

$$
(\Lambda(k) \zeta)_{v}=N_{v} f
$$

where $f$ corresponds to $\zeta$ as described above and $N_{v}$ was defined in Section 2. This map is a well known tool in the spectral theory of boundary value problems and has also been used in quantum graph theory [Ong 2006; Kuchment 2005].

The functions $\varphi_{v}$ and formulae for $\Lambda$. Given $v \in V$, let $\varphi_{v}$ be the function in $M(k)$ that satisfies

$$
\varphi_{v}(u)=\delta_{u v} \quad \text { for all } u, v \in V .
$$

The functions $\varphi_{v}$ are given by the following explicit expressions. Let $v \in e, e \in E^{\text {int }}$ and identify $e$ with $[0, \rho]$ where $v$ corresponds to the point 0 . Then

$$
\varphi_{v}(x)=\frac{\sin k(\rho-x)}{\sin k \rho}, \quad x \in[0, \rho]=e .
$$

In the same way, if $e \in E^{\text {ext }}$ and $v$ is identified with the point 0 , then

$$
\varphi_{v}(x)=e^{i k x}, \quad x \in[0, \infty)=e .
$$


If the dependence on $k$ needs to be emphasized, we will write $\varphi_{v}(x ; k)$ instead of $\varphi_{v}(x)$.

Lemma 4.1. If $k \in \mathbb{C}_{+}$then the map $\Lambda(k)$ is invertible. Its matrix entries are given by

$$
\begin{array}{ll}
\Lambda_{u v}=0 & \text { if } u \neq v, u \ngtr v ; \\
\Lambda_{u v}=\sum_{\substack{e \in E^{\text {int }} \\
u, v \in e}} \frac{k}{\sin k \rho(e)} & \text { if } u \neq v, u \sim v ; \\
\Lambda_{v v}=i k q(v)-k \sum_{\substack{e \in E^{\text {int }} \\
v \in e}} \cot k \rho(e) & \text { for any } v \in V ;
\end{array}
$$

where $q(v)$ was defined in Section 2.

Proof. If $\Lambda(k) \zeta=0$, then the corresponding function $f \in \mathcal{M}(k) \subset L^{2}(\varphi)$ satisfies the Kirchhoff boundary condition at every vertex, which implies that $f \in \operatorname{Dom} H$ and $H f=k^{2} f$. Since $\operatorname{Spec}(H)=[0, \infty)$ and $\operatorname{Im} k>0$, this implies that $f=0$. Therefore $\Lambda(k)$ is invertible.

By the definition of $\varphi_{v}$, we have

$$
\Lambda_{u v}=N_{u} \varphi_{v} .
$$

The formulae for the matrix entries are obtained by combining this with (4-1) and (4-2).

It follows from Lemma 4.1 that $\Lambda(k)$ can be extended to a meromorphic function of $k \in \mathbb{C}$ whose poles are all on the real axis, and that for any $u, v \in V$ one has

$$
\Lambda_{u v}(k)=\Lambda_{v u}(k) \quad \text { and } \quad \overline{\Lambda_{u v}(k)}=\Lambda_{u v}(-\bar{k}), \quad k \in \mathbb{C} .
$$

In the calculations below the expressions $\Lambda_{u v}^{-1}$ will denote the matrix entries of $(\Lambda(k))^{-1}$.

The complex-scaled version of $\varphi_{v}$. We will need a version of the functions $\varphi_{v}$ pertaining to the complexscaled operator $H(\varkappa)$. Let $k \in \mathbb{C}_{+}$and $\varkappa \in \mathbb{C}$ be such that $k e^{\varkappa} \in \mathbb{C}_{+}$. Given $v \in V$, we define $\varphi_{v}^{\varkappa}$ by

$$
\varphi_{v}^{\varkappa}(x ; k)= \begin{cases}\varphi_{v}(x ; k) & \text { if } x \in \mathscr{G}_{0} ; \\ \varphi_{v}(0 ; k) e^{\varkappa / 2} \exp \left(i k e^{\varkappa} x\right) & \text { if } x \in \ell=[0, \infty), \ell \in E^{\mathrm{ext}} .\end{cases}
$$

Clearly, $\varphi_{v}^{\varkappa}$ is a solution to the equation $H(\varkappa) \varphi_{v}^{\varkappa}=k^{2} \varphi_{v}^{\varkappa}$ on every edge of $\varphi_{\text {. It }}$ is also straightforward to see that $\varphi_{v}^{\varkappa} \in \widetilde{C}(\varphi)$ and $\varphi_{v}^{\varkappa}$ satisfies the boundary condition (2-5) on every external vertex. For $f \in \widetilde{C}(\mathscr{G})$, let us denote

$$
N_{v}^{\varkappa} f= \begin{cases}N_{v} f & \text { if } v \in V^{\text {int }} \\ N_{v}^{\text {int }} f+e^{-3 \varkappa / 2} N_{v}^{\text {ext }} f & \text { if } v \in V^{\text {ext }} .\end{cases}
$$

It is straightforward to see that

$$
\Lambda_{u v}=N_{u}^{\varkappa} \varphi_{v}^{\varkappa} \quad \text { for all } u, v \in V,
$$

where the left-hand side depends on $k$ but not on $\varkappa$. Moreover,

$$
\overline{\varphi_{v}^{\mathcal{x}}(x ; k)}=\varphi_{v}^{\bar{\varkappa}}(x ;-\bar{k}) .
$$


The resolvent identity. Let $H_{D}$ be the self-adjoint operator in $L^{2}(\varphi)$ defined by $H_{D} f=-f^{\prime \prime}$ with a Dirichlet boundary condition at every vertex of $\mathscr{G}$. Given $\varkappa \in \mathbb{C}$, we define the "complex-scaled" version of $H_{D}$ as follows; $H_{D}(\varkappa)$ is the operator acting in $L^{2}(\varphi)$ defined by

$$
\left(H_{D}(\varkappa) f\right)(x)= \begin{cases}-f^{\prime \prime}(x) & \text { if } x \in \mathscr{G}_{0}, \\ -e^{-2 \varkappa} f^{\prime \prime}(x) & \text { if } x \in \mathscr{G}_{\infty}\end{cases}
$$

with a Dirichlet boundary condition at every vertex of $\mathscr{G}$. Of course, $H_{D}(\varkappa)$ splits into an orthogonal sum of operators acting on $L^{2}(e)$ for all $e \in E$. We see immediately that in addition to its essential spectrum $e^{-2 \varkappa}[0, \infty)$, the operator $H_{D}(\varkappa)$ has a discrete set of positive eigenvalues with finite multiplicities.

We set

$$
R_{D}^{\varkappa}(k)=\left(H_{D}(\varkappa)-k^{2} I\right)^{-1}, \quad R^{\varkappa}(k)=\left(H(\varkappa)-k^{2} I\right)^{-1},
$$

whenever the inverse operators exist. We denote by $R^{\mathcal{x}}(k ; x, y)$, where $x, y \in \mathscr{G}$, the integral kernel of the resolvent $R^{\varkappa}(k)$; we define $R_{D}^{\varkappa}(k ; x, y)$ from $R_{D}^{\varkappa}(k)$ analogously.

The fact that $H_{D}(\varkappa)$ and $H(\varkappa)$ coincide except for different boundary conditions at each of the $|V|$ vertices indicates that the difference of the two resolvents should have rank $|V|$. Our next theorem makes this explicit. Formulae of this type are well known in the theory of boundary value problems; see [Gesztesy et al. 2009; 2007], for example. In the context of graphs, similar considerations have been used in [Kostrykin and Schrader 1999; 2006; Kostrykin et al. 2007; Ong 2006].

Theorem 4.2. For any $k \in \mathbb{C}_{+}$and any $\varkappa \in \mathbb{C}$, such that $k e^{\varkappa} \in \mathbb{C}_{+}$, we have

$$
R^{\varkappa}(k ; x, y)-R_{D}^{\varkappa}(k ; x, y)=-\sum_{u, v \in V} \Lambda_{u v}^{-1}(k) \varphi_{v}^{\varkappa}(x ; k) \varphi_{u}^{\varkappa}(y ; k)
$$

for any $x, y \in \mathscr{G}$.

Proof. 1. Let $\widetilde{R}^{\varkappa}(k)$ be the operator in $L^{2}(\mathscr{G})$ with the integral kernel given by

$$
\widetilde{R}^{\varkappa}(k ; x, y)=R_{D}^{\varkappa}(k ; x, y)-\sum_{u, v \in V} \Lambda_{u v}^{-1}(k) \varphi_{v}^{\varkappa}(x ; k) \varphi_{u}^{\varkappa}(y ; k) .
$$

We need to prove that $\widetilde{R}^{\varkappa}(k)$ is a bounded operator, that it maps $L^{2}(\varphi)$ into Dom $H(\varkappa)$ and that the identities

$$
\begin{aligned}
& \left(H(\varkappa)-k^{2} I\right) \widetilde{R}^{\varkappa}(k)=I \\
& \widetilde{R}^{\varkappa}(k)\left(H(\varkappa)-k^{2} I\right)=I
\end{aligned}
$$

hold true. First note that since $\varphi_{v}^{\varkappa}$ decays exponentially on all leads, the boundedness of $\widetilde{R}^{\varkappa}(k)$ is obvious. Next, using (4-6), (4-8) one obtains $\widetilde{R}^{\varkappa}(k)^{*}=\widetilde{R}^{\bar{\varkappa}}(-\bar{k})$. From here and (2-7) by taking adjoints we see that (4-11) is equivalent to

$$
\left(H(\bar{\varkappa})-(-\bar{k})^{2}\right) \widetilde{R}^{\bar{\varkappa}}(-\bar{k})=I
$$

which is (4-10) with $-\bar{k}, \bar{\varkappa}$ instead of $k, \varkappa$. We note that $k \in \mathbb{C}_{+}, k e^{\varkappa} \in \mathbb{C}_{+}$if and only if $-\bar{k} \in \mathbb{C}_{+}$, $-\bar{k} e^{\bar{x}} \in \mathbb{C}_{+}$. Thus, (4-11) follows from (4-10). 
2. It suffices to prove that for a dense set of elements $f \in L^{2}(\mathscr{G})$, the inclusion $\widetilde{R}^{\varkappa}(k) f \in \operatorname{Dom} H(\varkappa)$ and the identity

$$
\left(H(\varkappa)-k^{2} I\right) \widetilde{R}^{\varkappa}(k) f=f
$$

hold true. Let $f$ be from the dense set of all continuous functions compactly supported on $\varphi$ and vanishing near all vertices of $\varphi$. Let us check that the function $g=\widetilde{R}^{\varkappa}(k) f$ belongs to Dom $H(\varkappa)$. It is clear that the restriction of $g$ onto any edge $e$ of $\varphi$ belongs to the Sobolev space $W_{2}^{2}(e)$. Thus, it suffices to check that $g$ belongs to $\widetilde{C}(\varphi)$ and satisfies the boundary conditions (2-5) and (2-6).

Denote $g_{0}=R_{D}^{\varkappa}(k) f$. Since $g_{0} \in \operatorname{Dom} H_{D}(\varkappa), g_{0}$ vanishes on all vertices. Therefore $g_{0}$ lies in $\widetilde{C}(\varphi)$ and satisfies (2-5) at every external vertex $v$. As mentioned on page 742 , the functions $\varphi_{v}^{\varkappa}$ also belong to $\widetilde{C}(\mathscr{G})$ and satisfy (2-5) at every external vertex $v$. Thus, $g$ also has these properties.

Our next task is to prove that the boundary condition (2-6) is satisfied for the function $g$. Suppose that $f$ is supported on a single edge, which we identify with $[0, \rho]$. Then the integral kernel of $R_{D}^{\varkappa}(k)$ can be explicitly calculated, which gives

$$
g_{0}^{\prime}(0)=\int_{0}^{\rho} \frac{\sin k(\rho-x)}{\sin k \rho} f(x) d x .
$$

Similarly, if $f$ is supported on a lead $[0, \infty)$, then a direct calculation shows that

$$
g_{0}^{\prime}(0)=e^{2 \varkappa} \int_{0}^{\infty} \exp \left(i k e^{\varkappa} x\right) f(x) d x .
$$

Combining this, we see that for any $w \in V^{\text {ext }}$ we have

$$
N_{w}^{\varkappa} g_{0}=\int_{\varphi} f(x) \varphi_{w}^{\varkappa}(x) d x .
$$

Using the last identity and (4-7), for any $w \in V^{\text {ext }}$ we get:

$$
N_{w}^{\varkappa} g=\int_{\mathscr{G}} f(x) \varphi_{w}^{\varkappa}(x) d x-\sum_{u, v \in V} \Lambda_{u v}^{-1} \Lambda_{w v} \int_{\mathscr{G}} f(x) \varphi_{u}^{\varkappa}(x) d x=0,
$$

and so the boundary condition (2-6) is satisfied for $g$. Thus, $g \in \operatorname{Dom} H(\varkappa)$, as required.

3. It remains to note that the identity (4-12) follows from the fact that $R_{D}^{\varkappa}$ is the resolvent of $H_{D}(\varkappa)$ and the fact that $\varphi_{v}^{\varkappa}$ satisfies the equation $H(\varkappa) \varphi_{v}^{\varkappa}=k^{2} \varphi_{v}^{\varkappa}$ on every edge and lead of $\varphi$.

A trace formula. The trace formula (4-13) below results by calculating the traces of both sides of (4-9). Since the right-hand side of (4-9) is a finite rank operator, the trace is well defined; the fact that the value of (4-13) does not depend on $\varkappa$ can be proved by complex scaling, but the direct proof is almost as easy.

The identity (4-13) below can be rephrased by saying that the (modified) perturbation determinant of the pair of operators $H(\varkappa), H_{D}(\varkappa)$ equals det $\Lambda(k)$. Statements of this nature (for $\varkappa=0$ ) are well known in the theory of boundary value problems; see e.g. [Carron 2002] and references therein. The key to our proof of Theorem 3.1 will be (4-13) and Lemma 5.1, in which $\operatorname{det} A(k)$ and $\operatorname{det} \Lambda(k)$ are related. 
Theorem 4.3. For any $k \in \mathbb{C}_{+}$and any $\varkappa \in \mathbb{C}$, such that $k e^{\varkappa} \in \mathbb{C}_{+}$, we have

$$
\operatorname{Tr}\left(R^{\varkappa}(k)-R_{D}^{\varkappa}(k)\right)=-\frac{d}{d k} \operatorname{det} \Lambda(k) .
$$

In particular, the left-hand side is independent of $\varkappa$.

Proof. 1. Theorem 4.2 yields

$$
\operatorname{Tr}\left(R^{\mathcal{\varkappa}}(k)-R_{D}^{\varkappa}(k)\right)=-\sum_{u, v \in V} \Lambda_{u v}^{-1}(k) \sigma_{u v}^{\varkappa}(k),
$$

where

$$
\sigma_{u v}^{\varkappa}(k)=\int_{\varphi} \varphi_{u}^{\varkappa}(x ; k) \varphi_{v}^{\varkappa}(x ; k) d x .
$$

We next compute the coefficients $\sigma_{u v}$ explicitly. If $v \neq u$ and $v \not \chi u$ then $\operatorname{supp} \varphi_{v}^{\varkappa} \cap \operatorname{supp} \varphi_{u}^{\varkappa}=\varnothing$ and so $\sigma_{u v}=0$. If $v \neq u$ and $v \sim u$ then by (4-1)

$$
\sigma_{u v}=\sum_{\substack{e \in E^{\text {int }} \\ u, v \in e}} \int_{0}^{\rho} \frac{\sin k x}{\sin k \rho(e)} \frac{\sin k(\rho(e)-x)}{\sin k \rho(e)} d x=\frac{1}{2 k} \sum_{\substack{e \in E^{\text {int }} \\ u, v \in e}} \frac{\sin k \rho(e)-k \rho(e) \cos k \rho(e)}{(\sin k \rho(e))^{2}},
$$

and finally,

$$
\begin{aligned}
\sigma_{v v} & =\sum_{\substack{e \in E^{\text {int }} \\
v \in e}} \int_{0}^{\rho(e)}\left(\frac{\sin k x}{\sin k \rho(e)}\right)^{2} d x+q(v) \int_{0}^{\infty}\left(e^{\varkappa / 2} \exp \left(i k e^{\varkappa} x\right)\right)^{2} d x \\
& =\frac{1}{2 k} \sum_{\substack{e \in E^{\text {int }} \\
v \in e}} \frac{k \rho(e)-\cos k \rho(e) \sin k \rho(e)}{(\sin k \rho(e))^{2}}+\frac{i}{2 k} q(v) .
\end{aligned}
$$

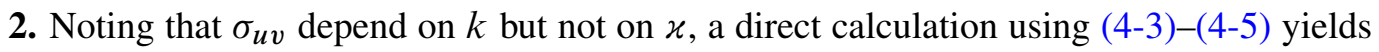

$$
\frac{1}{2 k} \frac{d}{d k} \Lambda_{u v}(k)=\sigma_{u v}(k) \text {. }
$$

It follows that

$$
\operatorname{Tr}\left(R^{\varkappa}(k)-R_{D}^{\varkappa}(k)\right)=-\sum_{u, v \in V} \Lambda_{u v}^{-1}(k) \frac{1}{2 k} \frac{d}{d k} \Lambda_{u v}(k)=-\frac{1}{2 k} \operatorname{Tr}\left(\Lambda^{-1}(k) \frac{d}{d k} \Lambda(k)\right)=-\frac{\frac{d}{d k} \operatorname{det} \Lambda(k)}{2 k \operatorname{det} \Lambda(k)},
$$

as required.

\section{Proof of Theorems 3.1 and 2.2}

Calculation of det $\boldsymbol{A}(\boldsymbol{k})$. Given $k \in \mathbb{C}$, we define

$$
\delta(k)=\prod_{e \in E^{\text {int }}}(k \sin k \rho(e)) .
$$

Let $A(k)$ be the matrix defined on page 736 . 
Lemma 5.1. For any $k \in \mathbb{C}_{+}$, we have the identity

$$
\operatorname{det} A(k)= \pm \frac{2^{\left|E^{\mathrm{int}}\right|} i^{\left|E^{\mathrm{int}}\right|-|V|}}{k^{\left|E^{\mathrm{int}}\right|+|V|}} \delta(k) \operatorname{det} \Lambda(k),
$$

where the sign \pm depends on the ordering of the rows and columns of the matrix $A(k)$.

Proof. 1. Let us order the rows and the columns of $A(k)$ in such a way that the first $|V|$ rows correspond to the conditions $N_{v}(u)=0$, and the first $|V|$ columns correspond to the variables $\zeta$. Then $A(k)$ can be written in the block form as

$$
A=\left(\begin{array}{rr}
0 & M \\
-N & P
\end{array}\right)
$$

where 0 is the $|V| \times|V|$ zero matrix and $P$ is a $\left(2\left|E^{\text {int }}\right|+\left|E^{\text {ext }}\right|\right) \times\left(2\left|E^{\text {int }}\right|+\left|E^{\text {ext }}\right|\right)$ matrix. The elements of $N$ are 0 or 1 , the elements of $M$ are $0, \pm 1, \pm e^{ \pm i k \rho}$, and the elements of $P$ are $0, \pm 1$, or $e^{ \pm i k \rho}$. For example, the matrix (3-4) is written in this form.

2. Let us reorder the rows of $P$ in such a way that any two constraints associated with the continuity conditions at the two endpoints of the same edge follow one another. Let us also reorder the columns of $P$ such that each variable $\beta_{e}$ follows the corresponding variable $\alpha_{e}$. For example, the block $P$ of the matrix (3-4) after such reordering will be

$$
\left(\begin{array}{ccccc}
1 & 1 & 0 & 0 & 0 \\
z_{1} & z_{1}^{-1} & 0 & 0 & 0 \\
0 & 0 & 1 & 1 & 0 \\
0 & 0 & z_{2} & z_{2}^{-1} & 0 \\
0 & 0 & 0 & 0 & 1
\end{array}\right) .
$$

In general, after this reordering, $P$ assumes a block-diagonal structure with blocks either of size $2 \times 2$ with elements

$$
\left(\begin{array}{cc}
1 & 1 \\
e^{i k \rho} & e^{-i k \rho}
\end{array}\right)
$$

or of size $1 \times 1$ with the element 1 . From here it follows that

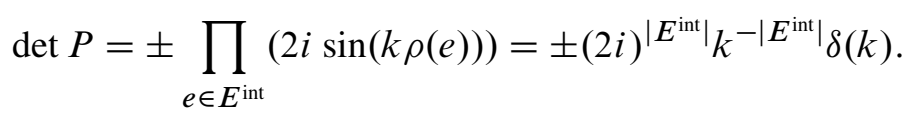

In particular, since $k \in \mathbb{C}_{+}$, the matrix $P$ is invertible.

3. By applying the Schur complement method to (5-3) one obtains

$$
\operatorname{det} A=\operatorname{det} P \operatorname{det}\left(M P^{-1} N\right) \text {. }
$$

Let us prove that

$$
i k M P^{-1} N=\Lambda(k) \text {. }
$$

Let $\zeta \in \mathbb{C}^{|V|}$ and let $a=P^{-1} N \zeta$. The vector $a$ represents a set of parameters $\alpha, \beta, \gamma$. Let $f \in \mathscr{L}(k)$ be the solution with this set of parameters. The equation $P a=N \zeta$ implies that the solution $f$ is continuous 
on $\mathscr{G}$ and satisfies $f(v)=\zeta_{v}$ for any vertex $v$. Next, the coordinates of the vector $i k M P^{-1} N \zeta=i k M a$ are given by

$$
i k(M a)_{v}=N_{v} f .
$$

This shows that $i k M a=\Lambda(k) \zeta$, as required.

4. By combining (5-4)-(5-6) one obtains

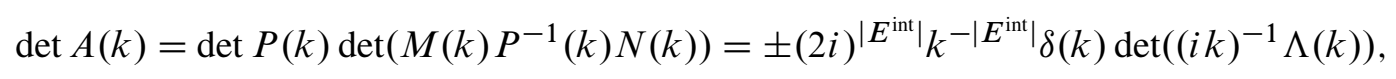

which yields (5-2) immediately.

Proof of Theorem 3.1. 1. Let $k \in \mathbb{C}_{+}$and let $\chi_{0}$ and $\chi_{\infty}$ be defined as in Section 2. Clearly, $\chi_{0} R_{D}(k) \chi_{0}$ is an orthogonal sum of resolvents of the operators $-d^{2} / d x^{2}$ on the intervals $(0, \rho(e)), e \in E^{\text {int }}$, with Dirichlet boundary conditions. For each such operator we have that $\left(-d^{2} / d x^{2}-k^{2}\right)^{-1}$ is trace class and

$$
\begin{aligned}
\operatorname{Tr}\left(-d^{2} / d x^{2}-k^{2}\right)^{-1} & =\sum_{n=0}^{\infty}\left((\pi n / \rho)^{2}-k^{2}\right)^{-1}=-\frac{1}{2 k^{2}}-\frac{1}{2 k} \sum_{n=-\infty}^{\infty} \frac{1}{k-\pi n / \rho} \\
& =-\frac{1}{2 k^{2}}-\frac{\rho}{2 k} \cot (k \rho)=-\frac{\frac{d}{d k}(k \sin (k \rho))}{2 k(k \sin (k \rho))} .
\end{aligned}
$$

Summing over all edges, a direct calculation shows that $\chi_{0} R_{D}(k) \chi_{0}$ is a trace class operator and

$$
\operatorname{Tr}\left(\chi_{0} R_{D}(k) \chi_{0}\right)=-\frac{\frac{d}{d k} \delta(k)}{2 k \delta(k)} .
$$

2. Let $k \in \mathbb{C}_{+}, k e^{\varkappa} \in \mathbb{C}_{+}$. It is easy to see that the resolvent $R_{D}^{\varkappa}(k)$ commutes with $\chi_{0}, \chi_{\infty}$ and that

$$
\chi_{0} R_{D}^{\varkappa}(k) \chi_{0}=\chi_{0} R_{D}(k) \chi_{0} .
$$

Therefore we have

$$
R^{\varkappa}(k)-\chi_{\infty} R_{D}^{\varkappa}(k) \chi_{\infty}=R^{\varkappa}(k)-R_{D}^{\varkappa}(k)+\chi_{0} R_{D}(k) \chi_{0} .
$$

By combining Theorem 4.3 and (5-8), we obtain

$$
\operatorname{Tr}\left(R^{\varkappa}(k)-\chi_{\infty} R_{D}^{\varkappa}(k) \chi_{\infty}\right)=-\frac{\frac{d}{d k} \operatorname{det} \Lambda(k)}{2 k \operatorname{det} \Lambda(k)}-\frac{\frac{d}{d k} \delta(k)}{2 k \delta(k)}=-\frac{\frac{d}{d k}(\delta(k) \operatorname{det} \Lambda(k))}{2 k \delta(k) \operatorname{det} \Lambda(k)} .
$$

Using Lemma 5.1, we then obtain

$$
\operatorname{Tr}\left(R^{\varkappa}(k)-\chi_{\infty} R_{D}^{\mathcal{\varkappa}}(k) \chi_{\infty}\right)=\frac{\left|E^{\mathrm{int}}\right|+|V|}{2 k^{2}}-\frac{\frac{d}{d k} \operatorname{det} A(k)}{2 k \operatorname{det} A(k)},
$$

for all $k \in \mathbb{C}_{+}$and $k e^{\varkappa} \in \mathbb{C}_{+}$.

3. The right-hand side of (5-10) is a single-valued meromorphic function of $k \in \mathbb{C}$. Let $\tau^{\mathscr{x}}(k)$ be the left-hand side of (5-10). For each fixed $\varkappa \in \mathbb{C}$, the function $\tau^{\varkappa}(k)$ is meromorphic in $\mathbb{C}$ with the cut along the line determined by the condition $k^{2} \in \sigma_{\text {ess }}(H(\varkappa))=e^{-2 \varkappa}[0, \infty)$. In other words, $\tau^{\varkappa}$ is meromorphic 
and single-valued in each of the two half-planes $\operatorname{Im} k e^{\varkappa}>0$ and $\operatorname{Im} k e^{\varkappa}<0$. By the uniqueness of analytic continuation, for each $\varkappa$ the identity (5-10) extends to all $k$ such that $\operatorname{Im} k e^{\varkappa}>0$.

4. Let $k_{0} \in \mathscr{R}$ with the algebraic multiplicity $m\left(k_{0}\right) \geq 1$ and let $\theta \in(0, \pi)$ with $-\theta<\arg k_{0} \leq 0$. Then $\operatorname{Im} k_{0} e^{i \theta}>0$ and so the identity (5-10) with $\varkappa=i \theta$ holds for all $k$ near $k_{0}$. If $\gamma$ is a sufficiently small circle with centre at $k_{0}$, then the multiplicity $m\left(k_{0}\right)$ equals the rank, or equivalently the trace, of the Riesz spectral projection

$$
P^{\theta}\left(k_{0}\right)=-\frac{1}{2 \pi i} \int_{\gamma} R^{i \theta}(k) 2 k d k
$$

Next, since the operator $H_{D}(i \theta)$ restricted to $L^{2}\left(\varphi_{\infty}\right)$ has no eigenvalues, the operator valued function $\chi_{\infty} R_{D}^{i \theta}(k) \chi_{\infty}$ is analytic for $\operatorname{Im} k e^{i \theta} \neq 0$. It follows that

$$
-\frac{1}{2 \pi i} \int_{\gamma} \chi_{\infty} R_{D}^{i \theta}(k) \chi_{\infty} 2 k d k=0 .
$$

By taking the trace of the difference of the last two equations and using (5-10) we obtain

$$
m\left(k_{0}\right)=-\frac{1}{2 \pi i} \int_{\gamma} \operatorname{Tr}\left(R^{i \theta}(k)-\chi_{\infty} R_{D}^{i \theta}(k) \chi_{\infty}\right) 2 k d k=\frac{1}{2 \pi i} \int_{\gamma} \frac{\frac{d}{d k} \operatorname{det} A(k)}{\operatorname{det} A(k)} d k .
$$

Therefore $m\left(k_{0}\right)$ equals the order of the zero of $\operatorname{det} A(k)$ at $k=k_{0}$, as required.

Proof of Theorem 2.2. This theorem is well known but we give its proof for completeness.

1. First note that by Theorem 4.2, the difference of the resolvents of $H(\varkappa)$ and $H_{D}(\varkappa)$ is a finite rank operator. By Weyl's theorem on the invariance of the essential spectrum under a relatively compact perturbation we obtain

$$
\sigma_{\mathrm{ess}}(H(\varkappa))=\sigma_{\mathrm{ess}}\left(H_{D}(\varkappa)\right)=e^{-2 \varkappa}[0, \infty) .
$$

2. The fact that the family $H(\varkappa)$ is analytic in the sense of Kato follows again from Theorem 4.2, since $H_{D}(\varkappa)$ is analytic in the sense of Kato and each of the functions $\varphi_{v}^{\varkappa}$ is analytic in $\varkappa$.

3. The identity (2-8) can be checked by a direct calculation.

4. Let $k \in \mathscr{R}$ and let $f$ be the corresponding eigenfunction. For any $\theta \in(0, \pi)$ with $-\theta<\arg k \leq 0$, let $f_{\theta}$ be the function defined formally by $f_{\theta}=U(i \theta) f$. More precisely, we set $f_{\theta}=f$ on $\varphi_{0}$ and

$$
f_{\theta}(x)=f(0) e^{i \theta / 2} \exp \left(i k e^{i \theta} x\right)
$$

for $x$ on any lead $\ell=[0, \infty)$. By the choice of $\theta$, we have $\operatorname{Im} k e^{i \theta}>0$ and so $f_{\theta} \in L^{2}(\varphi)$. A straightforward inspection shows that $f_{\theta} \in \operatorname{Dom} H(i \theta)$ and $H(i \theta) f_{\theta}=k^{2} f_{\theta}$.

5. Conversely, let $\lambda \notin e^{-2 i \theta}[0, \infty)$ be an eigenvalue of $H(i \theta)$ for $\theta \in(0, \pi)$. Write $\lambda=k^{2}$ with $\operatorname{Im} k e^{i \theta}>0$. Then, for the corresponding eigenfunction $g$ of $H(i \theta)$ we have $g(x)=g(0) \exp \left(i k e^{i \theta} x\right)$ on any lead of $\mathscr{G}$. A direct inspection shows that $g=f_{\theta}$ in the same sense as (5-12), where $f$ is a resonance eigenfunction. Thus, $k \in \mathscr{R}$ and in particular, $\operatorname{Im} k \leq 0$. It follows that $2 \pi-2 \theta<\arg k^{2} \leq 2 \pi$. 


\section{An example}

Here we consider resonances of a particular simple graph $\mathscr{G}(c)$, where $c \in[0,1]$ is a certain geometric parameter. The graph $\mathscr{G}(c)$ was also considered in [Exner and Lipovský 2010, Section 4], but with different boundary conditions at the vertices. The graph $\mathscr{G}(c)$ is Weyl for $c<1$ and non-Weyl for $c=1$. This section has two goals. The first one is to discuss the transition between the Weyl and the nonWeyl cases in order to throw new light on the failure of the Weyl law. Our second goal is to obtain rigorous bounds on the locations of individual resonances of $\mathscr{G}(c)$, which was not addressed by Exner and Lipovský.

Definition of $\mathscr{G}(\boldsymbol{c})$. Given $c \in[0,1)$, we consider the graph $\varphi_{0}(c)$ which consists of two vertices $v_{1}$ and $v_{2}$ and two edges $e_{1}=\left[0, \rho_{1}\right], \rho_{1}=(1-c) \pi$, and $e_{2}=\left[0, \rho_{2}\right], \rho_{2}=(1+c) \pi$. The vertex $v_{2}$ is identified with the point 0 of $e_{1}$ and with the point 0 of $e_{2}$, and the vertex $v_{1}$ is identified with the point $\rho_{1}$ of $e_{1}$ and with the point $\rho_{2}$ of $e_{2}$. Thus, the graph $\varphi_{0}(c)$ is simply a circle with the circumference vol $\mathscr{G}_{0}(c)=2 \pi$ for all $c$. We attach a lead $\ell_{1}$ at $v_{1}$ and a lead $\ell_{2}$ at $v_{2}$ and denote the thus extended graph by $\mathscr{G}(c)$. Geometrically, $\mathscr{G}(c)$ is a circle with two leads attached to it. Finally, for $c=1$, let $\mathscr{G}(c)$ be the circle of length $2 \pi$ with two leads attached at the same point.

We will denote by $H(c)$ the operator $-d^{2} / d x^{2}$ acting in $L^{2}(\mathscr{G}(c))$ subject to the usual continuity and Kirchhoff boundary conditions at the vertices $v_{1}$ and $v_{2}$. By Theorem 1.2, the graph $\mathscr{G}(c)$ is Weyl if and only if $c<1$. At the same time, the graph $\mathscr{G}(1)$ can be regarded as the limit of $\mathscr{G}(c)$ as $c \rightarrow 1$ in an obvious geometric sense, so we need to explain what happens to resonances as $c \rightarrow 1$. As we will see, roughly speaking, a half of the resonances of $H(c)$ move off to infinity as $c \rightarrow 1$. We will obtain bounds on the curves along which the resonances move as $c$ increases from 0 to 1 .

The matrix $A(k, c)$ for $\mathscr{G}(\boldsymbol{c})$. Let us display the constraints (3-3) corresponding to the graph $\mathscr{G}(c)$; the matrix $A(k, c)$ will be built up of the rows corresponding to these constraints. We denote $z_{j}=e^{i k \rho_{j} / 2}$, $j=1,2$. The constraints corresponding to the vertex $v_{1}$ are

$$
\begin{aligned}
\alpha_{1} z_{1}^{2}+\beta_{1} z_{1}^{-2}-\zeta_{1} & =0, \\
\alpha_{2} z_{2}^{2}+\beta_{2} z_{2}^{-2}-\zeta_{1} & =0, \\
\gamma_{1}-\zeta_{1} & =0, \\
-\alpha_{1} z_{1}^{2}+\beta_{1} z_{1}^{-2}-\alpha_{2} z_{2}^{2}+\beta_{2} z_{2}^{-2}+\gamma_{1} & =0 .
\end{aligned}
$$

The first three lines are the continuity conditions, and the last is the requirement that the sum of the outgoing derivatives vanishes. Similarly, the constraints corresponding to the vertex $v_{2}$ are

$$
\begin{aligned}
\alpha_{1}+\beta_{1}-\zeta_{2} & =0 \\
\alpha_{2}+\beta_{2}-\zeta_{2} & =0 \\
\gamma_{2}-\zeta_{2} & =0 \\
\alpha_{1}-\beta_{1}+\alpha_{2}-\beta_{2}+\gamma_{2} & =0
\end{aligned}
$$


We list these constraints in the order $R_{1}, R_{5}, R_{2}, R_{6}, R_{3}, R_{7}, R_{4}, R_{8}$, and order the variables as $\alpha_{1}$, $\beta_{1}, \alpha_{2}, \beta_{2}, \gamma_{1}, \gamma_{2}, \zeta_{1}, \zeta_{2}$. This leads to the matrix

$$
A(k, c)=\left(\begin{array}{ccccccrr}
z_{1}^{2} & z_{1}^{-2} & 0 & 0 & 0 & 0 & -1 & 0 \\
1 & 1 & 0 & 0 & 0 & 0 & 0 & -1 \\
0 & 0 & z_{2}^{2} & z_{2}^{-2} & 0 & 0 & -1 & 0 \\
0 & 0 & 1 & 1 & 0 & 0 & 0 & -1 \\
0 & 0 & 0 & 0 & 1 & 0 & -1 & 0 \\
0 & 0 & 0 & 0 & 0 & 1 & 0 & -1 \\
-z_{1}^{2} & z_{1}^{-2} & -z_{2}^{2} & z_{2}^{-2} & 1 & 0 & 0 & 0 \\
1 & -1 & 1 & -1 & 0 & 1 & 0 & 0
\end{array}\right)
$$

Calculation of det $\boldsymbol{A}(\boldsymbol{k}, \boldsymbol{c})$. The graph $\mathscr{G}(c)$ has a reflection symmetry with respect to the midpoints of $e_{1}$ and $e_{2}$. This allows to decompose the space $\mathscr{L}(k)$ into the direct sum of the subspaces corresponding to even and odd functions with respect to this symmetry. We use this decomposition to represent the matrix $A(k, c)$ in a block-diagonal form where the blocks correspond to the even and odd solutions. More precisely, let

$$
T_{1}=\left(\begin{array}{cccccccc}
1 & 1 & 0 & 0 & 0 & 0 & 0 & 0 \\
0 & 0 & 1 & 1 & 0 & 0 & 0 & 0 \\
0 & 0 & 0 & 0 & 1 & 1 & 0 & 0 \\
0 & 0 & 0 & 0 & 0 & 0 & 1 & 1 \\
1 & -1 & 0 & 0 & 0 & 0 & 0 & 0 \\
0 & 0 & 1 & -1 & 0 & 0 & 0 & 0 \\
0 & 0 & 0 & 0 & 1 & -1 & 0 & 0 \\
0 & 0 & 0 & 0 & 0 & 0 & 1 & -1
\end{array}\right), \quad T_{2}=\left(\begin{array}{cccccccc}
z_{1}^{-1} & 0 & 0 & 0 & z_{1}^{-1} & 0 & 0 & 0 \\
z_{1} & 0 & 0 & 0 & -z_{1} & 0 & 0 & 0 \\
0 & z_{2}^{-1} & 0 & 0 & 0 & z_{2}^{-1} & 0 & 0 \\
0 & z_{2} & 0 & 0 & 0 & -z_{2} & 0 & 0 \\
0 & 0 & 1 & 0 & 0 & 0 & 1 & 0 \\
0 & 0 & 1 & 0 & 0 & 0 & -1 & 0 \\
0 & 0 & 0 & 1 & 0 & 0 & 0 & 1 \\
0 & 0 & 0 & 1 & 0 & 0 & 0 & -1
\end{array}\right) .
$$

A straightforward calculation shows that $\operatorname{det} T_{1}=\operatorname{det} T_{2}=16$. Next, let $\widetilde{A}(k, c)=T_{1} A(k, c) T_{2}$; the reader is invited to check that the matrix $\widetilde{A}(k)$ can be written as

$$
\tilde{A}=2\left(\begin{array}{cc}
\tilde{A}_{\text {even }} & 0 \\
0 & \tilde{A}_{\text {odd }}
\end{array}\right),
$$

with blocks

$$
\tilde{A}_{\text {even }}=\left(\begin{array}{cccc}
2 C_{1} & 0 & 0 & -1 \\
0 & 2 C_{2} & 0 & -1 \\
0 & 0 & 1 & -1 \\
-2 i S_{1} & -2 i S_{2} & 1 & 0
\end{array}\right), \quad \tilde{A}_{\text {odd }}=\left(\begin{array}{cccc}
2 i S_{1} & 0 & 0 & -1 \\
0 & 2 i S_{2} & 0 & -1 \\
0 & 0 & 1 & -1 \\
-2 C_{1} & -2 C_{2} & 1 & 0
\end{array}\right),
$$

where we have used the notation $C_{j}=\cos \left(k \rho_{j} / 2\right), S_{j}=\sin \left(k \rho_{j} / 2\right), j=1,2$. Straightforward calculations of $\operatorname{det}\left(\tilde{A}_{\text {even }}\right)$ and $\operatorname{det}\left(\tilde{A}_{\text {odd }}\right)$ now yield

Theorem 6.1. For all $k \in \mathbb{C}$ and all $c \in[0,1)$ one has

$$
\operatorname{det} A(k, c)=4 F_{\text {even }}(k, c) F_{\text {odd }}(k, c),
$$


where

$$
\begin{gathered}
F_{\text {even }}(k, c)=i \cos (k c \pi)+i \cos (k \pi)+2 \sin (k \pi), \\
F_{\text {odd }}(k, c)=i \cos (k c \pi)-i \cos (k \pi)-2 \sin (k \pi) .
\end{gathered}
$$

We will call the zeros of $F_{\text {even }}(\cdot, c)$ (resp. of $\left.F_{\text {odd }}(\cdot, c)\right)$ the even (resp. odd) resonances. It is not difficult to check that the resonance eigenfunctions which correspond to even/odd resonances are even/odd with respect to the symmetry of the graph $\mathscr{G}(c)$. By Theorem 2.3, the real even/odd resonances are actually eigenvalues of $H(c)$ and therefore we will call them even/odd eigenvalues.

Finally, it is not difficult to check that the resonances of $H(1)$ are given, as expected, by the zeros of $\operatorname{det} A(k, 1)$. In fact, in this case we have $F_{\text {odd }}(k, 1)=-2 \sin (k \pi)$ and

$$
F_{\text {even }}(k, 1)=2 i e^{-i k \pi} \neq 0 \text { for all } k \in \mathbb{C} .
$$

Thus, the resonances of $H(1)$ coincide with the solutions to $\sin (k \pi)=0$, i.e., they are given by $k \in \mathbb{Z}$. By Theorem 2.3, these resonances (for $k \neq 0$ ) coincide with the eigenvalues of $H(1)$ and all of them have multiplicity one. This shows that for $c=1$ we have the asymptotics (1-3) with $W=\pi=\frac{1}{2}$ vol $\mathscr{G}_{0}$.

\section{Locating the odd resonances.}

Theorem 6.2. (i) For any $c \in[0,1]$, any $n \in \mathbb{Z}$ and any $y \geq 0$ one has $F_{\text {odd }}\left(n+\frac{1}{2}-i y, c\right) \neq 0$.

(ii) For any $c \in[0,1]$ and any $k=x-i y$ with $y>|x| / \sqrt{3}$ one has $F_{\text {odd }}(k, c) \neq 0$.

Proof. (i) By an explicit calculation,

$$
F_{\text {odd }}\left(n+\frac{1}{2}-i y, c\right)=i \cos \left(\left(n+\frac{1}{2}-i y\right) \pi c\right)+(-1)^{n} \sinh (y \pi)-2(-1)^{n} \cosh (y \pi)=A+B,
$$

where

$$
\begin{aligned}
|A| & =\left|\cos \left(\left(n+\frac{1}{2}-i y\right) \pi c\right)\right| \\
& =\left|\cos \left(\left(n+\frac{1}{2}\right) \pi c\right) \cosh (y \pi c)+i \sin \left(\left(n+\frac{1}{2}\right) \pi c\right) \sinh (y \pi c)\right| \\
& \leq \cosh (y \pi c) \leq \cosh (y \pi)
\end{aligned}
$$

and

$$
|B|=2 \cosh (y \pi)-\sinh (y \pi)=\cosh (y \pi)+\mathrm{e}^{-y \pi} .
$$

We deduce that

$$
\left|F_{\text {odd }}\left(n+\frac{1}{2}-i y, c\right)\right| \geq|B|-|A| \geq \mathrm{e}^{-y \pi}>0 .
$$

(ii) We start by observing that $\left|F_{\text {odd }}(k, c)\right| \geq 2 A-B$ where

$$
A=|\sin (k \pi)|, \quad B=|\cos (k \pi)-\cos (k \pi c)|=\left|\int_{c}^{1} k \pi \sin (k \pi s) d s\right| .
$$

If $u \in \mathbb{R}$ and $v \geq 0$ then

$$
\sin (u-i v)=\sin (u) \cosh (v)-i \cos (u) \sinh (v) .
$$


Therefore

$$
\sinh (v) \leq|\sin (u-i v)| \leq \cosh (v) .
$$

We deduce that $A \geq \sinh (y \pi)$ and

$$
B \leq \int_{c}^{1}|k| \pi \cosh (y \pi s) d s=\frac{|k|}{y}(\sinh (y \pi)-\sinh (y \pi s)) \leq \frac{|k|}{y} \sinh (y \pi) .
$$

These bounds imply that $2 A-B>0$ if $2 y>|k|$, which yields the theorem immediately.

It follows that all odd resonances are located in the rectangles

$$
\Pi_{n}^{\text {odd }}=\left\{x-i y:|x-n|<\frac{1}{2}, 0 \leq y \leq \frac{2|n|+1}{2 \sqrt{3}}\right\}, \quad n \in \mathbb{Z} .
$$

The following statement, in combination with Rouche's theorem, shows that each of the rectangles $\Pi_{n}^{\text {odd }}$ contains exactly one odd resonance of algebraic multiplicity one for all $c \in[0,1]$.

Theorem 6.3. If $c=0$ there is a resonance of algebraic multiplicity one at $k=n-i \log (3) / \pi$ for every odd $n \in \mathbb{Z}$ and an eigenvalue of multiplicity one at $k=n$ for every nonzero even $n \in \mathbb{Z}$. There is also a resonance of algebraic multiplicity one at $k=0$. No other odd resonances or eigenvalues exist if $c=0$.

The proof follows from the explicit formula

$$
F_{\text {odd }}(k, 0)=\frac{i}{2}\left(e^{i k \pi}+3\right)\left(1-e^{-i k \pi}\right) .
$$

By the implicit function theorem, we obtain that each of the zeros of $F_{\text {odd }}(\cdot ; c)$ is a real analytic function of $c \in[0,1]$ with values in $\Pi_{n}^{\text {odd }}$. The set of all odd resonances for all such $c$ is therefore the union of a sequence of bounded real analytic curves.

It is interesting to note that each of these resonance curves intersects the real axis, thereby (by Theorem 2.3) giving rise to embedded eigenvalues. This happens at rational values of $c$. More precisely, a direct computation shows that $F_{\text {odd }}(k, c)=0$ for $k \in \mathbb{R}$ if and only if

$$
k=m+n \quad \text { and } \quad c=\frac{m-n}{m+n} \quad \text { for some } m, n \in \mathbb{N} .
$$

Figure 1 plots a typical odd resonance curve as $c$ increases from 0 to 1 . It starts at $7-i \log (3) / \pi$, when $c=0$. The curve then passes through 7 when $c=\frac{1}{7}, \frac{3}{7}, \frac{5}{7}, 1$.

\section{Locating the even resonances.}

Theorem 6.4. (i) For any $c \in[0,1]$, any $n \in \mathbb{Z}$ and any $y \geq 0$ one has $F_{\mathrm{even}}\left(n+\frac{1}{2}-i y, c\right) \neq 0$.

(ii) For any $c \in[0,1)$ and any $k=x-i y$ with $y>\frac{\log 3}{\pi(1-|c|)}$, one has $F_{\text {odd }}(k, c) \neq 0$.

Proof. (i) We have

$$
F_{\text {even }}\left(n+\frac{1}{2}-i y, c\right)=A-B,
$$

where $A, B$ are as in the proof of Theorem 6.2(i). The rest of the proof is the same as in Theorem 6.2(i). 


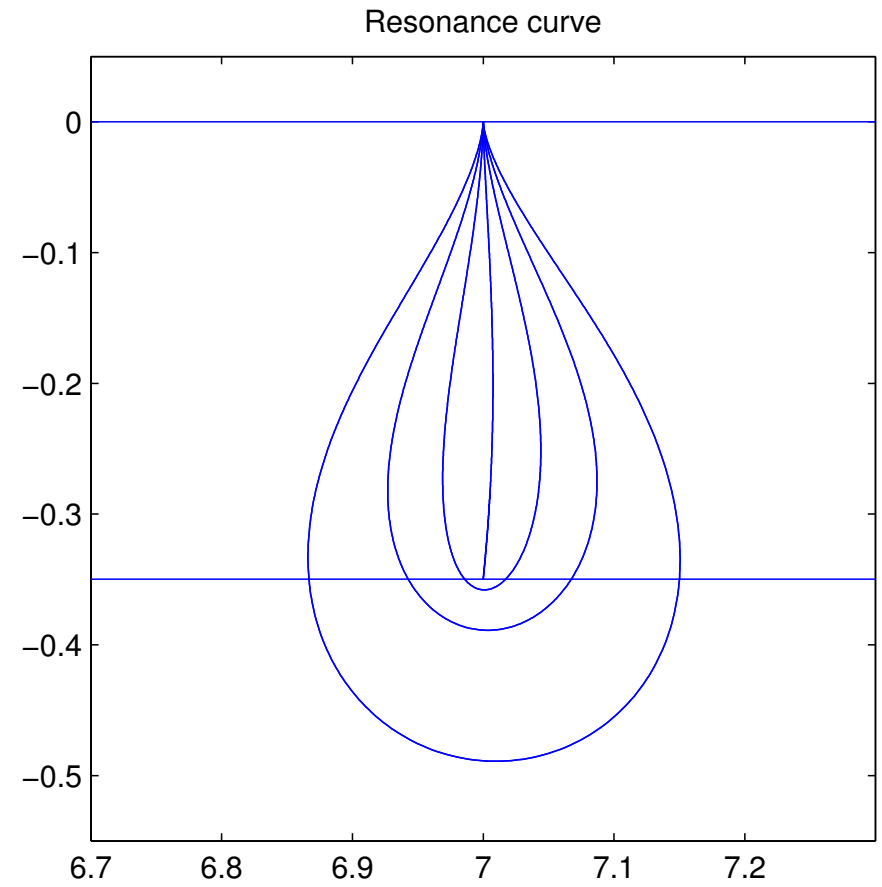

Figure 1. The odd resonance curve in $\Pi_{7}^{\text {odd }}$.

(ii) For any $k=x-i y$ we have

$$
\begin{aligned}
\frac{1}{2} \mathrm{e}^{y \pi|c|}+\frac{1}{2} & \geq \cosh (y \pi c) \geq|\cos (x \pi c) \cosh (y \pi c)+i \sin (x \pi c) \cosh (y \pi c)| \\
& \geq|\cos (x \pi c) \cosh (y \pi c)+i \sin (x \pi c) \sinh (y \pi c)|=|\cos (k \pi c)|
\end{aligned}
$$

and

$$
|i \cos (k \pi)+2 \sin (k \pi)| \geq \frac{1}{2}\left|\mathrm{e}^{i k \pi}\right|-\frac{3}{2}\left|\mathrm{e}^{-i k \pi}\right|=\frac{1}{2} \mathrm{e}^{y \pi}-\frac{3}{2} \mathrm{e}^{-y \pi} .
$$

Now suppose $F_{\text {even }}(k, c)=0$; then $\cos (k \pi c)=-i \cos (k \pi)-2 \sin (k \pi)$ and therefore, combining (6-2) and (6-3), we obtain

$$
\mathrm{e}^{y \pi} \leq \mathrm{e}^{y \pi|c|}+1+3 \mathrm{e}^{-y \pi} .
$$

If $y \geq \log (3) / \pi$ or equivalently $\mathrm{e}^{y \pi} \geq 3$ then

$$
\mathrm{e}^{y \pi} \leq \mathrm{e}^{y \pi|c|}+2 \leq \mathrm{e}^{y \pi|c|}+\frac{2}{3} \mathrm{e}^{y \pi} .
$$

A simple manipulation then yields that $y \leq \frac{\log 3}{\pi(1-|c|)}$, and the required result follows.

It follows that for $c \in[0,1)$ the even resonances are located in the rectangles

$$
\Pi_{n}^{\text {even }}(c)=\left\{x+i y:|x-n|<\frac{1}{2}, 0 \leq y \leq \frac{\log 3}{\pi(1-|c|)}\right\} .
$$




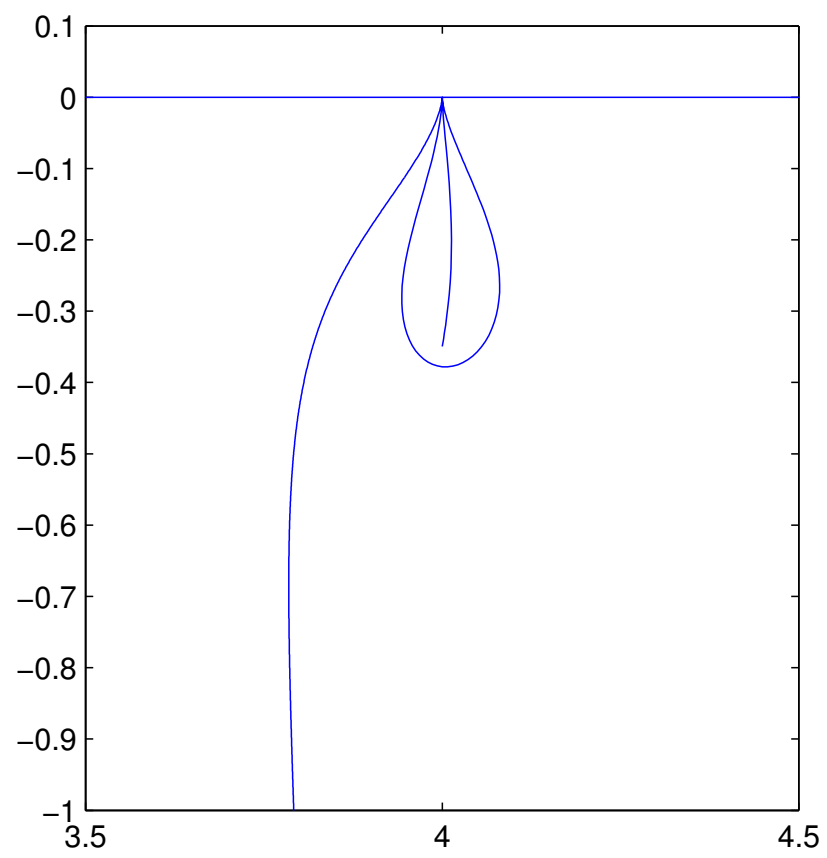

Figure 2. The even resonance curve in $\Pi_{4}^{\text {even }}$.

Just as in the odd case, the following statement shows that for each $n \in \mathbb{Z}$ and $c \in[0,1)$, the rectangle $\Pi_{n}^{\text {even }}(c)$ contains exactly one resonance.

Theorem 6.5. If $c=0$ there is an even resonance of the algebraic multiplicity one at $k=n-i \log (3) / \pi$ for every even $n \in \mathbb{Z}$ and an even eigenvalue of multiplicity one at $k=n$ for every nonzero odd $n \in \mathbb{Z}$. There are no other even resonances.

The proof follows from the explicit formula

$$
F_{\text {even }}(k, 0)=-\frac{1}{2} i\left(e^{i k \pi}-3\right)\left(1+e^{-i k \pi}\right) .
$$

Just as in the odd case, we obtain that the resonances are given by branches of real analytic functions of $c \in[0,1)$ with values in $\Pi_{n}^{\text {even }}(c)$. However, in contrast with the odd case, the height of the rectangles $\Pi_{n}^{\text {even }}(c)$ is not uniformly bounded in $c$. Moreover:

Theorem 6.6. Let $n \in \mathbb{Z}$ and let $k_{n}=k_{n}(c)$ be the unique solution to $F_{\mathrm{even}}(k, c)=0$ with $k_{n}(c) \in$ $\Pi_{n}^{\text {even }}(c)$. Then $\operatorname{Im} k_{n}(c) \rightarrow-\infty$ as $c \rightarrow 1$.

Proof. Suppose that the conclusion of the theorem is false. Then there exists a sequence $c_{m} \rightarrow 1$ such that $\operatorname{Im} k_{n}\left(c_{m}\right)$ is bounded. By passing to a subsequence we can assume that $k_{n}\left(c_{m}\right) \rightarrow k_{n}^{\infty} \in \mathbb{C}$ as $m \rightarrow \infty$. This would imply that $F_{\text {even }}\left(k_{n}^{\infty}, 1\right)=0$ by the joint continuity of the function $F_{\text {even }}$. This is impossible by $(6-1)$. 
Therefore, all even resonances move off to infinity and this explains the failure of the Weyl law for $c=1$. Formal calculations and numerical analysis suggest that the rate of divergence of $\operatorname{Im} k_{n}(c)$ as $c \rightarrow 1$ is logarithmic.

As in the odd case, the even resonance curves intersect the real axis for some rational values of $k$. A direct computation shows that $F_{\text {even }}(k, c)=0$ for $k \in \mathbb{R}$ if and only if

$$
k=m+n-1 \quad \text { and } \quad c=\frac{m-n}{m+n-1} \quad \text { for some } n, m \in \mathbb{N} .
$$

Figure 2 plots a typical even resonance curve as $c$ increases from 0 to 1 . It starts at $4-i \log (3) / \pi$ when $c=0$. The curve then passes through 4 when $c=\frac{1}{5}, \frac{3}{5}$ and diverges to $\infty$ as $c \rightarrow 1$.

\section{References}

[Aguilar and Combes 1971] J. Aguilar and J. M. Combes, "A class of analytic perturbations for one-body Schrödinger Hamiltonians", Comm. Math. Phys. 22 (1971), 269-279. MR 49 \#10287 Zbl 0219.47011

[Borthwick 2010] D. Borthwick, "Sharp upper bounds on resonances for perturbations of hyperbolic space", Asymptot. Anal. 69:1-2 (2010), 45-85. MR 2012a:58052 Zbl 05831996

[Carron 2002] G. Carron, "Déterminant relatif et la fonction Xi", Amer. J. Math. 124:2 (2002), 307-352. MR 1890995 (2003c:58023)

[Christiansen 2004] T. Christiansen, "Asymptotics for a resonance-counting function for potential scattering on cylinders", $J$. Funct. Anal. 216:1 (2004), 172-190. MR 2005j:35162 Zbl 0752.35046

[Davies 2003] E. B. Davies, "Eigenvalues of an elliptic system”, Math. Z. 243:4 (2003), 719-743. MR 2004b:35248 Zbl 1032. 34078

[Davies and Incani 2010] E. B. Davies and P. A. Incani, "Spectral properties of matrices associated with some directed graphs", Proc. Lond. Math. Soc. (3) 100:1 (2010), 55-90. MR 2010m:65091 Zbl 1189.15011

[Davies et al. 2010] E. B. Davies, P. Exner, and J. Lipovský, "Non-Weyl asymptotics for quantum graphs with general coupling conditions", J. Phys. A 43:47 (2010), 474013, 16. MR 2011m:81112 Zbl 1204.81078

[Exner and Lipovský 2007] P. Exner and J. Lipovský, "Equivalence of resolvent and scattering resonances on quantum graphs", pp. 73-81 in Adventures in mathematical physics (Cergy-Pontoise, 2006), edited by F. Germinet et al., Contemp. Math. 447, Amer. Math. Soc., Providence, RI, 2007. MR 2009k:81247 Zbl 1137.81010

[Exner and Lipovský 2010] P. Exner and J. Lipovský, "Resonances from perturbations of quantum graphs with rationally related edges", J. Phys. A 43:10 (2010), Art. id 105301. MR 2011e:81102

[Froese 1997] R. Froese, "Asymptotic distribution of resonances in one dimension", J. Differential Equations 137:2 (1997), 251-272. MR 98f:81339 Zbl 0955.35057

[Gesztesy et al. 2007] F. Gesztesy, M. Mitrea, and M. Zinchenko, "Variations on a theme of Jost and Pais", J. Funct. Anal. 253:2 (2007), 399-448. MR 2008k:35081 Zbl 1133.47010

[Gesztesy et al. 2009] F. Gesztesy, M. Mitrea, and M. Zinchenko, "On Dirichlet-to-Neumann maps and some applications to modified Fredholm determinants", pp. 191-215 in Methods of spectral analysis in mathematical physics, edited by J. Janas et al., Oper. Theory Adv. Appl. 186, Birkhäuser, Basel, 2009. MR 2012b:47053 Zbl 1169.47014

[Incani 2009] P. A. Incani, Spectral theory of directed graphs, Ph.D. thesis, King's College, London, 2009.

[Korotyaev 2005] E. Korotyaev, "Inverse resonance scattering on the real line", Inverse Problems 21:1 (2005), 325-341. MR 2006a:34033 Zbl 1074.34081

[Kostrykin and Schrader 1999] V. Kostrykin and R. Schrader, "Kirchhoff's rule for quantum wires", J. Phys. A 32:4 (1999), 595-630. MR 99m:81280 Zbl 0928.34066

[Kostrykin and Schrader 2006] V. Kostrykin and R. Schrader, "Laplacians on metric graphs: eigenvalues, resolvents and semigroups", pp. 201-225 in Quantum graphs and their applications, Contemp. Math. 415, Amer. Math. Soc., Providence, RI, 2006. MR 2007j:34041 Zbl 1122.34066 
[Kostrykin et al. 2007] V. Kostrykin, J. Potthoff, and R. Schrader, "Heat kernels on metric graphs and a trace formula", pp. 175198 in Adventures in mathematical physics, Contemp. Math. 447, Amer. Math. Soc., Providence, RI, 2007. MR 2010b:81163 Zbl 1155.34017

[Kottos and Smilansky 2003] T. Kottos and U. Smilansky, "Quantum graphs: a simple model for chaotic scattering”, J. Phys. A 36:12 (2003), 3501-3524. MR 2004g:81064 Zbl 1038.81031

[Kuchment 2004] P. Kuchment, "Quantum graphs, I: Some basic structures”, Waves Random Media 14:1 (2004), S107-S128. Special section on quantum graphs. MR 2005h:81148

[Kuchment 2005] P. Kuchment, "Quantum graphs, II: Some spectral properties of quantum and combinatorial graphs”, J. Phys. A 38:22 (2005), 4887-4900. MR 2006a:81035

[Kuchment 2008] P. Kuchment, "Quantum graphs: an introduction and a brief survey", pp. 291-312 in Analysis on graphs and its applications, Proc. Sympos. Pure Math. 77, Amer. Math. Soc., Providence, RI, 2008. MR 2010b:81058 Zbl 1210.05169

[Langer 1931] R. E. Langer, "On the zeros of exponential sums and integrals", Bull. Amer. Math. Soc. 37:4 (1931), 213-239. MR 1562129 Zbl 0001.34403

[Moreno 1973] C. J. Moreno, "The zeros of exponential polynomials, I", Compositio Math. 26 (1973), 69-78. MR 47 \#7007 Zbl 0267.33001

[Ong 2006] B.-S. Ong, "On the limiting absorption principle and spectra of quantum graphs", pp. 241-249 in Quantum graphs and their applications, Contemp. Math. 415, Amer. Math. Soc., Providence, RI, 2006. MR 2008d:81073

[Parnovski 1995] L. B. Parnovski, "Spectral asymptotics of Laplace operators on surfaces with cusps", Math. Ann. 303:2 (1995), 281-296. MR 97a:11080 Zbl 0849.35093

[Reed and Simon 1978] M. Reed and B. Simon, Methods of modern mathematical physics, IV: Analysis of operators, Academic Press, New York, 1978. MR 58 \#12429c

[Simon 1973] B. Simon, "Resonances in $n$-body quantum systems with dilatation analytic potentials and the foundations of time-dependent perturbation theory", Ann. of Math. (2) 97 (1973), 247-274. MR 50 \#6378 Zbl 0252.47009

[Sjöstrand and Zworski 1991] J. Sjöstrand and M. Zworski, "Complex scaling and the distribution of scattering poles", J. Amer. Math. Soc. 4:4 (1991), 729-769. MR 92g:35166 Zbl 0752.35046

[Stefanov 2006] P. Stefanov, "Sharp upper bounds on the number of the scattering poles", J. Funct. Anal. 231:1 (2006), 111142. MR 2006i:35267 Zbl 1099.35074

[Zworski 1987] M. Zworski, "Distribution of poles for scattering on the real line", J. Funct. Anal. 73:2 (1987), $277-296$. MR 88h:81223 Zbl 0662.34033

Received 22 Mar 2010. Revised 2 Aug 2010. Accepted 14 Sep 2010.

E. BRIAN DAVIES: E.Brian.Davies@kcl.ac.uk

Department of Mathematics, King's College London, Strand, London WC2R 2LS, United Kingdom

http://www.kcl.ac.uk/nms/depts/mathematics/people/atoz/daviesb.aspx

ALEXANDER PUSHNITSKI: alexander.pushnitski@kcl.ac.uk

Department of Mathematics, King's College London, Strand, London WC2R 2LS, United Kingdom

http://www.mth.kcl.ac.uk/staff/a_pushnitski.html 


\title{
Analysis \& PDE
}

\author{
msp.berkeley.edu/apde
}

EDITORS

EDITOR-IN-CHIEF

Maciej Zworski

University of California

Berkeley, USA

BOARD OF EDITORS

\begin{tabular}{|c|c|c|c|}
\hline Michael Aizenman & $\begin{array}{l}\text { Princeton University, USA } \\
\text { aizenman@math.princeton.edu }\end{array}$ & Nicolas Burq & $\begin{array}{l}\text { Université Paris-Sud 11, France } \\
\text { nicolas.burq@math.u-psud.fr }\end{array}$ \\
\hline Luis A. Caffarelli & $\begin{array}{l}\text { University of Texas, USA } \\
\text { caffarel@math.utexas.edu }\end{array}$ & un-Yung Alice Chang & $\begin{array}{l}\text { Princeton University, USA } \\
\text { chang@math.princeton.edu }\end{array}$ \\
\hline Michael Christ & $\begin{array}{l}\text { University of California, Berkeley, USA } \\
\text { mchrist@math.berkeley.edu }\end{array}$ & Charles Fefferman & $\begin{array}{l}\text { Princeton University, USA } \\
\text { cf@math.princeton.edu }\end{array}$ \\
\hline Ursula Hamenstaedt & $\begin{array}{l}\text { Universität Bonn, Germany } \\
\text { ursula@math.uni-bonn.de }\end{array}$ & Nigel Higson & $\begin{array}{l}\text { Pennsylvania State Univesity, USA } \\
\text { higson@ math.psu.edu }\end{array}$ \\
\hline Vaughan Jones & $\begin{array}{l}\text { University of California, Berkeley, USA } \\
\text { vfr@math.berkeley.edu }\end{array}$ & Herbert Koch & $\begin{array}{l}\text { Universität Bonn, Germany } \\
\text { koch@math.uni-bonn.de }\end{array}$ \\
\hline Izabella Laba & $\begin{array}{l}\text { University of British Columbia, Canada } \\
\text { ilaba@math.ubc.ca }\end{array}$ & Gilles Lebeau & $\begin{array}{l}\text { Université de Nice Sophia Antipolis, France } \\
\text { lebeau@unice.fr }\end{array}$ \\
\hline László Lempert & $\begin{array}{l}\text { Purdue University, USA } \\
\text { lempert@math.purdue.edu }\end{array}$ & Richard B. Melrose & $\begin{array}{l}\text { Massachussets Institute of Technology, USA } \\
\text { rbm@math.mit.edu }\end{array}$ \\
\hline Frank Merle & $\begin{array}{l}\text { Université de Cergy-Pontoise, France } \\
\text { Frank.Merle@u-cergy.fr }\end{array}$ & William Minicozzi II & $\begin{array}{l}\text { Johns Hopkins University, USA } \\
\text { minicozz@ math.jhu.edu }\end{array}$ \\
\hline Werner Müller & $\begin{array}{l}\text { Universität Bonn, Germany } \\
\text { mueller@math.uni-bonn.de }\end{array}$ & Yuval Peres & $\begin{array}{l}\text { University of California, Berkeley, USA } \\
\text { peres@stat.berkeley.edu }\end{array}$ \\
\hline Gilles Pisier & $\begin{array}{l}\text { Texas A\&M University, and Paris } 6 \\
\text { pisier@math.tamu.edu }\end{array}$ & Tristan Rivière & $\begin{array}{l}\text { ETH, Switzerland } \\
\text { riviere@math.ethz.ch }\end{array}$ \\
\hline Igor Rodnianski & $\begin{array}{l}\text { Princeton University, USA } \\
\text { irod@math.princeton.edu }\end{array}$ & Wilhelm Schlag & $\begin{array}{l}\text { University of Chicago, USA } \\
\text { schlag@math.uchicago.edu }\end{array}$ \\
\hline Sylvia Serfaty & $\begin{array}{l}\text { New York University, USA } \\
\text { serfaty@ cims.nyu.edu }\end{array}$ & Yum-Tong Siu & $\begin{array}{l}\text { Harvard University, USA } \\
\text { siu@math.harvard.edu }\end{array}$ \\
\hline Terence Tao & $\begin{array}{l}\text { University of California, Los Angeles, USA } \\
\text { tao@math.ucla.edu }\end{array}$ & A Michael E. Taylor & $\begin{array}{l}\text { Univ. of North Carolina, Chapel Hill, USA } \\
\text { met@math.unc.edu }\end{array}$ \\
\hline Gunther Uhlmann & $\begin{array}{l}\text { University of Washington, USA } \\
\text { gunther@math.washington.edu }\end{array}$ & András Vasy & $\begin{array}{l}\text { Stanford University, USA } \\
\text { andras@math.stanford.edu }\end{array}$ \\
\hline Virgil Voiculescu & $\begin{array}{l}\text { University of California, Berkeley, USA } \\
\text { dvv@math.berkeley.edu }\end{array}$ & Steven Zelditch & $\begin{array}{l}\text { Northwestern University, USA } \\
\text { zelditch@math.northwestern.edu }\end{array}$ \\
\hline
\end{tabular}

\section{PRODUCTION}

contact@msp.org

Silvio Levy, Scientific Editor

Sheila Newbery, Senior Production Editor

See inside back cover or msp.berkeley.edu/apde for submission instructions.

The subscription price for 2011 is US \$120/year for the electronic version, and \$180/year for print and electronic. Subscriptions, requests for back issues from the last three years and changes of subscribers address should be sent to Mathematical Sciences Publishers, Department of Mathematics, University of California, Berkeley, CA 94720-3840, USA.

Analysis \& PDE, at Mathematical Sciences Publishers, Department of Mathematics, University of California, Berkeley, CA 94720-3840 is published continuously online. Periodical rate postage paid at Berkeley, CA 94704, and additional mailing offices.

APDE peer review and production are managed by EditFLOW ${ }^{\mathrm{TM}}$ from Mathematical Sciences Publishers.

PUBLISHED BY

mathematical sciences publishers

http://msp.org/

A NON-PROFIT CORPORATION

Typeset in IATEX

Copyright $(2011$ by Mathematical Sciences Publishers 


\section{ANALYSIS \& PDE}

\section{Volume $4 \quad$ No. $5 \quad 2011$}

Periodic solutions of nonlinear Schrödinger equations: a paradifferential approach JEAN-MARC DELORT

Standing ring blowup solutions for cubic nonlinear Schrödinger equations IAN ZWIERS

Non-Weyl resonance asymptotics for quantum graphs

E. BRIAN DAVIES and AlEXANDER Pushnitski

Improved lower bounds for Ginzburg-Landau energies via mass displacement 